Cassi R. Paslick · Alex N. Halliday

Rebecca A. Lange · Dodie James · J. Barry Dawson

\title{
Indirect crustal contamination: evidence from isotopic and chemical disequilibria in minerals from alkali basalts and nephelinites from northern Tanzania
}

\begin{abstract}
Alkali basalts and nephelinites from the volcanic province of northern Tanzania contain pyroxene and nepheline that show evidence for chemical and/or isotopic disequilibria with their host magmas. Olivine, pyroxene, nepheline and plagioclase all appear to be partially xenocrystic in origin. Five whole rock/mineral separate pairs have been analyzed for $\mathrm{Sr}, \mathrm{Nd}$, and $\mathrm{Pb}$ isotopic compositions. The ${ }^{206} \mathrm{~Pb} /{ }^{204} \mathrm{~Pb}$ ratios are distinct by as much as 20.94 (whole rock) vs. 19.10 (clinopyroxene separate). The $\mathrm{Sr}$ and $\mathrm{Nd}$ isotopic disequilibria vary from insignificant in the case of nepheline, to $\Delta{ }^{87} \mathrm{Sr} /{ }^{86} \mathrm{Sr}$ of 0.0002 and $\Delta \varepsilon_{\mathrm{Nd}}$ of 0.7 in the case of clinopyroxene. The mineral chemistry of 25 samples indicates the ubiquitous presence of minerals that did not crystallize from a liquid represented by the host rock. The northern Tanzanian magmas are peralkaline and exhibit none of the xenocrystic phases expected from crustal assimilation. The disequilibria cannot be the result of mantle source variations. Rather the xenocrystic phases present appear to have been derived from earlier alkali basaltic rocks or magmas that were contaminated by the crust. Material from this earlier magma was then mixed with batches of magma that subsequently erupted on the surface. Disequilibrium in volcanic rocks has potentially serious consequences for the use of whole rock data to identify source reservoirs. However, mass balance calculations reveal that the ${ }^{206} \mathrm{~Pb} /{ }^{204} \mathrm{~Pb}$ isotopic compositions of the erupted lavas were changed by less than $0.25 \%$ as a result of this indirect crustal contamination.
\end{abstract}

C.R. Paslick $(-)$

Physical Science Division, Rock Valley College,

Rockford, IL 61114, USA

A.N. Halliday $\cdot$ R.A. Lange

Department of Geological Sciences,

The University of Michigan, Ann Arbor, MI 48109-1063, USA

D. James · J.B. Dawson

Department of Geology and Geophysics,

University of Edinburgh, Edinburgh, EH9 3JW, UK

Editorial responsibility: I.S.E. Carmichael

\section{Introduction}

During the past several decades, isotopic data have been used to identify source reservoirs of volcanic rocks, particularly in oceanic areas where contamination by crustal material is minimal (Hart et al. 1986). On the continents, however, the effects of crustal contamination often dominate the isotopic composition of the magmas and it is difficult to discern the isotopic composition of the source (Carlson et al. 1981). Nonetheless, whole rock isotopic compositions have been used extensively to distinguish between contaminated and pristine continental volcanic rocks (DePaolo and Wasserburg 1979; Hawkesworth and Vollmer 1979; Lum et al. 1989). The presence of mantle xenoliths in some basalts is taken to indicate minimal crustal contamination because direct and rapid transport to the surface is implied (Spera 1984). Isotopic and trace element compositions of continental basalts have been used to distinguish between source reservoirs in the asthenosphere and continental lithosphere (Halliday et al. 1988, 1990; Fitton et al. 1991; Kempton et al. 1991; Paslick et al. 1995).

Despite the apparent success of geochemical studies on mafic continental volcanic rocks, they commonly display evidence for disequilibrium in both the isotopic and chemical composition of the minerals (Cortini and van Calsteren 1985; Simonetti and Bell 1994). Disequilibrium may have serious consequences for the value of whole rock isotopic data in identifying source reservoirs. Tests of the reliability of whole rock isotopic data for continental volcanic rocks have been undertaken through microanalysis of mineral phases and determination of $\mathrm{Sr}$, $\mathrm{Nd}$, and $\mathrm{Pb}$ isotopic compositions on whole rock/mineral separate pairs from volcanic rocks of northern Tanzania (Fig. 1). In a previous paper on this province (Paslick et al. 1995) whole rock isotopic compositions of those samples believed to be the least evolved $(\mathrm{MgO}>4 \mathrm{wt} \%)$ coupled with trace element data were used to identify the continental lithospheric mantle as the source reservoir of the volcanic rocks in northern Tanzania. In this paper, new mineral chemistry data are combined with isotopic 


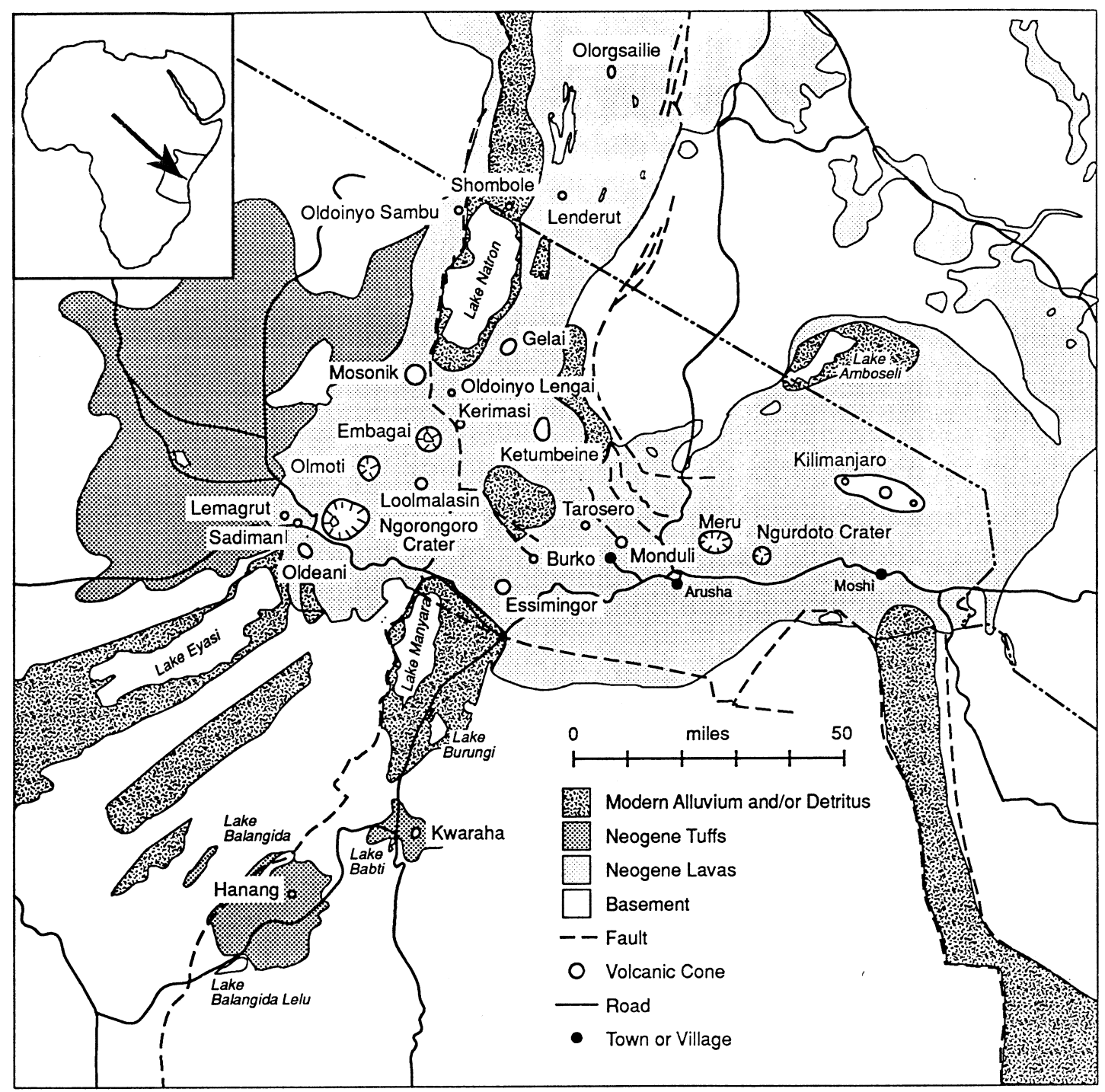

Fig. 1 Map of volcanic province of northern Tanzania (after Dawson 1992)

analyses on additional samples, including several evolved phonolites and five whole-rock/mineral separate pairs.

\section{Geologic background}

The volcanic province of northern Tanzania is the southern extension of the East African Rift and is superimposed on two major geologic provinces. The basement rock of western Tanzania is comprised of the Tanzanian shield, an Archean craton (Cahen et al. 1984), whereas the Mozambique Belt, a late-Proterozoic mobile belt that has been reworked during several orogenic events, is the basement rock of eastern Tanzania (Key et al. 1989). The present-day rift valley in northern Tanzania is a result of major faulting at ca. 1.2 Ma and was imposed on a broad tectonic depression that was the result of mid-Tertiary faulting and uplift of the Lake Victoria and Masai blocks (Dawson 1992). The earliest volcanic activity, beginning at about $8 \mathrm{Ma}$ (Bagdasaryan et al. 1973), followed the mid-Tertiary faulting event and consisted mainly of large volumes of alkali basalt, trachyte, and phonolite, erupted from shield volcanoes. Several small nephelinite volcanoes were also active during this time (i.e., Essimingor, Mosonik, and Sadiman; Dawson 1992). Volcanic activity following the 1.2 Ma faulting consisted of nephelinite, phonolite, and carbonatite magmas that erupted explosively from small centers (Dawson 1992).

In contrast to the narrow graben in Kenya (Baker et al. 1978), the rift valley in northern Tanzania is a broad depression trending north-south (Dawson 1992) (Fig. 1). It is bounded on the west by a major east facing escarpment that runs from Lake Natron in the north to Lake Balangida in the south. This scarp cuts through the eastern flanks of Loolmalasin, Ngorongoro, and Oldoinyo Sambu volcanoes. There is no eastern equivalent to this western escarpment; the eastern margin of the depression is formed by a series of small faults that are a southerly continuation of the Ngong-Turoka Fault in Kenya. Many minor faults occur on the floor of the depression (Fig. 1). We have sampled nine volcanoes (Burko, Hanang, Ketumbeine, Lemagrut, Ngorongoro, Meru, Monduli, Mosonik and Sadiman) and several small isolated cones.

\section{Analytical methods}

Mineral analyses were obtained on a Cameca Camebax electron microprobe configured with TAP, PET and LIF crystals and four 
spectrometers. Operating conditions for all mineral phases except titanite and perovskite were an accelerating potential of $15 \mathrm{kV}$ and a sample current of $10 \mathrm{nA}$. For analyses of titanite and perovskite a sample current of $40 \mathrm{nA}$ was used for $\mathrm{Ba}, \mathrm{Nb}, \mathrm{La}, \mathrm{Ce}, \mathrm{Pr}, \mathrm{Nd}, \mathrm{Sm}$, and $\mathrm{Gd}$; the remaining elements were determined at a sample current of $10 \mathrm{nA}$. A focused beam was used for olivine, clinopyroxene, kaersutite, titanite, perovskite, melanite, and spinel analyses. Feldspar, nepheline and phlogopite were scanned with a $6 \mu \mathrm{m}^{2}$ beam in order to minimize alkali migration. A counting time of $30 \mathrm{~s}$ was used for all elements in all phases with the exception of $\mathrm{F}$ in titanite, perovskite and phlogopite $(50 \mathrm{~s})$. Analytical data were corrected using the Cameca PAP program.

Sample powders were prepared by crushing in a tungsten-carbide shatterbox and were analyzed for major and trace elements by X-ray fluorescence spectroscopy (XRF) at Edinburgh University using methods outlined elsewhere (Fitton and Dunlop 1985).

Sample preparation and isotopic techniques have been described previously (Halliday et al. 1989, 1990). Isotopic compositions were measured on a VG Sector thermal ionization mass spectrometer equipped with six Faraday collectors. The $\mathrm{Sr}$ and Nd isotopic compositions were measured in multi-dynamic mode. The average ${ }^{143} \mathrm{Nd} /{ }^{144} \mathrm{Nd}$ ratio for the La Jolla standard was $0.511845 \pm 9(2 \sigma, \mathrm{n}=8)$. The average ${ }^{87} \mathrm{Sr} /{ }^{66} \mathrm{Sr}$ ratio for the NIST standard SRM 987 was $0.710250+15(2 \sigma, n=15)$. The $\mathrm{Sr}$ and Nd measurements were normalized for mass discrimination to ${ }^{86} \mathrm{Sr} /$ ${ }^{88} \mathrm{Sr}=0.1194$ and ${ }^{146} \mathrm{nd} /{ }^{144} \mathrm{nd}=0.7219$ respectively. The $\mathrm{Pb}$ isotopic compositions were measured in static mode and were corrected for fractionation and mass discrimination $(0.1 \%$ per a.m.u.) based on replicate analyses of NIST standard SRM 981.

\section{Results}

\section{Petrography}

Of the 100 samples collected, 25 were selected for analysis as representative of both the volcanoes sampled and the chemical variation of the suite. Two samples (C93-3 and L93-2M) are from small cones, located just south of Arusha. All samples except L93-2, MD93-7 and NG93-6 are nepheline normative. Olivine and pyroxene are ubiquitous phenocrysts, with the exception of two samples from Mosonik (MO93-1\&5) that contain only nepheline, titanite, and/or perovskite. One sample contains microphenocrysts of phlogopite (C93-3) and one sample contains melanite (B93-1). The two most evolved samples analyzed are NG93-6 and L93-2, with $\mathrm{SiO}_{2}$ of 65 and $57 \mathrm{wt} \%$ respectively. Sample NG93-6 contains phenocrysts of fayalitic olivine $\left(\mathrm{Fo}_{10}\right)$, a hedenbergitic pyroxene, and alkali-feldspar $\left(\mathrm{Or}_{30} \mathrm{Ab}_{67} \mathrm{An}_{3}\right)$. Sample L932 contains olivine $\left(\mathrm{FO}_{59}\right)$, augite, hornblende (with thick reaction rims) and plagioclase $\left(\mathrm{An}_{40}\right)$. All but two of the samples have a crystalline groundmass consisting of pyroxene, nepheline \pm oxides or pyroxenes, plagioclase \pm oxides. Olivine occurs in the groundmass of three samples. The groundmass in sample M93-4 is a welded, devitrified ash with crystallites of plagioclase; the groundmass in MO93-5 consists of green glass with crystallites of nepheline. The alteration state of the groundmass is variable. Slight chloritization and/or oxidation of the groundmass is common and there are several samples with areas of secondary calcite. The crystalline groundmass in samples G93-1, 2 and 3 is extensively cloritized, whereas the green glass in MO93-5 is very fresh.

\section{Mineralogy}

\section{Olivine}

Olivine occurs in fifteen of the analyzed samples. Representative olivine analyses are reported in Table 1 for samples with homogeneous populations. Core compositions for the olivines reported in Table 1 range from $\mathrm{Fo}_{90}$ to
Table 1 Representative olivine analyses

\begin{tabular}{|c|c|c|c|c|c|c|c|c|}
\hline Wt\% oxide & C93-3 & G93-1 & K93-1 & L93-2 & MD93-4 & MD93-7 & NG93-6 & OE93-4 \\
\hline \multicolumn{9}{|l|}{ Core } \\
\hline $\mathrm{SiO}_{2}$ & 38.78 & 37.80 & 36.43 & 35.08 & 41.02 & 39.95 & 29.89 & 38.86 \\
\hline $\mathrm{Al}_{2} \mathrm{O}_{3}$ & 0.04 & 0.03 & 0.04 & 0.03 & 0.01 & 0.05 & 0.01 & 0.04 \\
\hline $\mathrm{Cr}_{2} \mathrm{O}_{3}$ & 0.01 & 0.00 & 0.04 & 0.00 & 0.00 & 0.05 & 0.00 & 0.03 \\
\hline $\mathrm{FeO}$ & 17.38 & 20.59 & 27.66 & 35.40 & 9.66 & 11.23 & 60.98 & 17.02 \\
\hline $\mathrm{MnO}$ & 0.25 & 0.31 & 0.37 & 0.93 & 0.13 & 0.13 & 3.01 & 0.23 \\
\hline $\mathrm{NiO}$ & 0.21 & 0.19 & 0.12 & 0.00 & 0.39 & 0.24 & 0.01 & 0.22 \\
\hline $\mathrm{MgO}$ & 42.36 & 40.42 & 34.64 & 28.33 & 48.77 & 47.54 & 3.65 & 42.84 \\
\hline $\mathrm{CaO}$ & 0.22 & 0.42 & 0.24 & 0.25 & 0.06 & 0.20 & 0.58 & 0.31 \\
\hline Total & 99.25 & 99.74 & 99.54 & 100.03 & 100.04 & 99.39 & 98.11 & 99.55 \\
\hline $\mathrm{Fo}^{\mathrm{a}}$ & 81 & 78 & 69 & 59 & 90 & 88 & 10 & 82 \\
\hline \multicolumn{9}{|l|}{ Rim } \\
\hline $\mathrm{SiO}_{2}$ & relict & 38.06 & 35.52 & 35.12 & 40.43 & 37.78 & 30.00 & 36.69 \\
\hline $\mathrm{Al}_{2} \mathrm{O}_{3}$ & cores & 0.04 & 0.06 & 0.01 & 0.04 & 0.06 & 0.01 & 0.04 \\
\hline $\mathrm{Cr}_{2} \mathrm{O}_{3}$ & only & 0.00 & 0.07 & 0.00 & 0.13 & 0.01 & 0.00 & 0.04 \\
\hline $\mathrm{FeO}$ & & 20.96 & 33.87 & 35.13 & 12.66 & 22.27 & 61.47 & 26.31 \\
\hline $\mathrm{MnO}$ & & 0.27 & 0.52 & 1.00 & 0.17 & 0.34 & 3.01 & 0.53 \\
\hline $\mathrm{NiO}$ & & 0.17 & 0.07 & 0.03 & 0.32 & 0.15 & 0.02 & 0.06 \\
\hline $\mathrm{MgO}$ & & 39.78 & 29.11 & 27.93 & 45.94 & 39.05 & 3.68 & 34.77 \\
\hline $\mathrm{CaO}$ & & 0.39 & 0.36 & 0.26 & 0.29 & 0.23 & 0.57 & 0.32 \\
\hline Total & & 99.67 & 99.58 & 99.48 & 99.98 & 99.88 & 98.75 & 98.77 \\
\hline $\mathrm{Fo}^{\mathrm{a}}$ & & 77 & 61 & 59 & 87 & 76 & 10 & 70 \\
\hline
\end{tabular}

${ }^{\text {a }}$ Fo\% calulated via structural formulae on basis of four oxygens 
$\mathrm{Fo}_{59}$, with the exception of the very fayalitic olivine $\left(\mathrm{Fo}_{10}\right)$ in sample NG93-6. For those samples that have heterogeneous populations of olivine, for which there is no representative analysis, core and rim Fo contents have been plotted in Fig. 2 to illustrate the range in both composition and zoning. These samples contain both normally and reversely zoned olivines; there are several samples with unzoned olivine. In general the olivines are fractured and rounded, and show various degrees of alteration that include chlorite/epidote and iddingsite rims. In $\mathrm{C} 93-3$, olivine $\left(\mathrm{Fo}_{81}\right)$ has been almost completely replaced by iddingsite. Although iddingsite behaves optically as a single crystal in this sample, as described by Brown and Stephen (1959), back-scattered electron micrographs clearly show that the material is polycrystalline.

In sample MD93-4 there are two distinct populations of olivine crystals. One population is unzoned with compositions of $\mathrm{Fo}_{93}$. Olivines of $\mathrm{Fo}_{90-87}$ in the same sample are embayed and show signs of being stressed with development of subgrains and/or undulose extinction.

\section{Pyroxene}

Twenty-one samples contain augite as a phenocryst phase. Representative clinopyroxene analyses for samples with homogeneous populations are reported in Table 2. For those samples with heterogeneous populations of pyroxenes, for which there is no representative analysis, we have plotted core and rim compositions to illustrate the variability in the clinopyroxene population (Fig. 2). For this figure we have plotted $\mathrm{Al}^{\mathrm{iv}}$ as a measure of the exchange between the $\mathrm{Mg}-\mathrm{Si}$ and $\mathrm{Ti}-\mathrm{Al}^{\mathrm{iv}}$ components to illustrate zoning.

Several samples contain clinopyroxene with a significant acmite component. Sample NG93-6 contains a hedenbergite-rich pyroxene as well as augite. Sample MD93-4 contains rare orthopyroxene $\left(\mathrm{En}_{88}\right)$ with a crystallized rim of clinopyroxene $\left(\mathrm{En}_{30}\right)$, and small crystals of olivine $\left(\mathrm{Fo}_{79}\right)$. One of the enstatite grains contains a chromite inclusion. The pyroxenes show many different textural varieties, but most commonly the crystals are subhedral to euhedral and show very little alteration. Many of the augites show the concentric zoning and anomalous interference colors characteristic of titanaugite. Some phenocrysts contain substantial quantities of $\mathrm{TiO}_{2}$ and $\mathrm{Al}_{2} \mathrm{O}_{3}$ (Table 2).

\section{Nepheline}

Nepheline is the only feldspathoid present; it occurs as a phenocryst phase in seven of the samples. Representative analyses of phenocrysts are presented in Table 3 . The $\mathrm{K}_{2} \mathrm{O}$ content varies from 4 to $8 \mathrm{wt} \%$. The nephelines are generally euhedral, ranging in size from about 1 to $4 \mathrm{~mm}$. Nepheline crystals in B93-1, L93-3 and MO93-5 are very fresh. In the remaining samples the crystals are rounded and show some alteration around the edges and along fractures. The $\mathrm{Fe}_{2} \mathrm{O}_{3}$ of the nephelines in this study is slightly lower than those of nepheline phenocrysts from Shombole and Oldoinyo L'engai (Peterson 1989). Peterson reported oscillatory zoning in large nephelines from Shombole volcano in Kenya, visible as small changes in refractive index and birefringence. The nephelines in this study are not zoned with the exception of large nephelines from MO93-5, where zoning is visible as small changes in retardation colors as the mineral goes to extinction. However, this zoning is not detectable in the chemical analyses.

\section{Feldspar}

Feldspar occurs as a phenocryst phase in five of the samples. Representative analyses are presented in Table 4. Three samples contain plagioclase $\left(\mathrm{An}_{70}\right.$ to $\left.\mathrm{An}_{40}\right)$, whereas one sample, NG93-6, contains an alkali feldspar $\left(\mathrm{An}_{3} \mathrm{Ab}_{60} \mathrm{Or}_{37}\right)$. No zoning is detectable with the microprobe, although $\mathrm{SrO}$ and $\mathrm{BaO}$ concentrations are variable (0.20-0.75 and $0-0.40 \mathrm{wt} \%$ respectively). Texturally the feldspars are rounded with fuzzy edges; several show the fine-grained alteration to sericite common to plagioclase. In L93-2 plagioclase occurs both individually and in star-like clumps. Sample MD93-4 contains one plagioclase crystal $\left(\mathrm{An}_{45}\right)$ that is anhedral and most likely a xenocryst.

\section{Titanite, perovskite and melanite}

Titanite occurs in six foidites (Table 5). In three of the six samples (L93-3, MR93-2 and S93-2), titanite occurs as a phenocryst $(0.5$ to $3 \mathrm{~mm})$. Perovskite $(<0.1 \mathrm{~mm}$; Table 5 ) occurs as coarse grains in the groundmass of three of the six samples that contain titanite (MO93-1, MO93-5 and MR93-2). In MO93-1, perovskite occurs included in a titanite. Texturally, the titanites occur as euhedral (L93-3 and S93-2) and anhedral (B93-1, MO93-1, MO93-5 and MR93-2) crystals. Perovskite crystals are generally euhedral. Rare earth element concentrations are higher in perovskite than in titanite, similar to that found in rocks from Oldoinyo Lengai (Dawson et al. 1994).

Melanite garnet (titanian andradite) occurs in B93-1 (Table 6) and contains abundant pyroxene inclusions, as well as several included titanites. Two sets of representative analyses, normalized to 8 and 3 cations, are presented in Table 6. Analytical totals remained slightly low, even with the addition of several minor elements. For this reason we have attempted to estimate the water content of the garnets by normalizing the formula to 3 cations, assuming the Si site is 3 and forcing the $\mathrm{Y}$ site to 2 . Any excess cations in the $\mathrm{Y}$ site are assigned as $\mathrm{Al}^{\mathrm{iv}}$. Calculating a formula in this way gives a non-unique solution. Most of the analyses did not have enough Al to equal the excess in the $\mathrm{Y}$ site, suggesting the presence of $\mathrm{Fe}^{3+}$ in 
Table 2 Representative pyroxene analyses

\begin{tabular}{|c|c|c|c|c|c|c|c|c|c|c|c|c|c|}
\hline Wt\% oxide & C93-3 & G93-1 & H93-3 & K93-W3 & L93-2 & L93-3 & M93-4 & MD93-4 & MD93-7 & MR93-2 & NG93-6 & NG93-6 & RW93-6 \\
\hline \multicolumn{14}{|l|}{ Core } \\
\hline $\mathrm{SiO}_{2}$ & 49.09 & 51.02 & 52.98 & 47.27 & 50.59 & 48.90 & 48.35 & 58.73 & 52.53 & 50.20 & 47.81 & 50.49 & 47.30 \\
\hline $\mathrm{TiO}_{2}^{2}$ & 1.90 & 1.37 & 0.60 & 2.10 & 1.16 & 0.48 & 1.89 & 0.07 & 0.50 & 1.71 & 0.73 & 0.90 & 1.68 \\
\hline $\mathrm{Al}_{2} \mathrm{O}_{3}$ & 3.47 & 2.66 & 1.27 & 6.17 & 2.63 & 1.31 & 3.87 & 0.53 & 1.99 & 1.51 & 0.33 & 1.31 & 3.71 \\
\hline $\mathrm{Cr}_{2} \mathrm{O}_{3}$ & 0.33 & 0.00 & 0.63 & 0.10 & 0.00 & 0.00 & 0.32 & 0.13 & 0.30 & 0.00 & 0.00 & 0.00 & 0.13 \\
\hline $\mathrm{Fe}_{2} \mathrm{O}_{3}$ & 4.84 & 1.76 & 2.29 & 3.30 & 1.68 & 8.53 & 3.86 & 0.00 & 0.91 & 6.99 & 3.62 & 1.22 & 6.21 \\
\hline $\mathrm{FeO}$ & 1.99 & 6.90 & 1.86 & 4.46 & 7.78 & 16.34 & 5.54 & 4.34 & 7.61 & 5.43 & 20.74 & 11.85 & 1.82 \\
\hline $\mathrm{MnO}$ & 0.23 & 0.35 & 0.08 & 0.20 & 0.30 & 0.77 & 0.48 & 0.09 & 0.16 & 0.57 & 1.14 & 0.58 & 0.06 \\
\hline $\mathrm{MgO}$ & 14.05 & 13.44 & 16.12 & 12.66 & 13.18 & 2.47 & 11.73 & 34.59 & 18.71 & 10.82 & 4.25 & 12.22 & 13.78 \\
\hline $\mathrm{NiO}$ & 0.24 & 0.00 & 0.05 & 0.00 & 0.02 & 0.00 & 0.32 & 0.08 & 0.00 & 0.00 & 0.00 & 0.00 & 0.02 \\
\hline $\mathrm{CaO}$ & 23.65 & 22.16 & 23.25 & 22.06 & 21.42 & 17.89 & 21.72 & 0.21 & 16.20 & 21.34 & 19.53 & 19.36 & 23.19 \\
\hline $\mathrm{Na}_{2} \mathrm{O}$ & 0.56 & 0.57 & 0.73 & 0.63 & 0.54 & 3.13 & 0.96 & 0.10 & 0.30 & 1.93 & 0.72 & 0.47 & 0.41 \\
\hline Total & 100.37 & 100.24 & 99.86 & 98.95 & 99.30 & 99.79 & 99.03 & 98.86 & 99.22 & 100.49 & 98.86 & 98.40 & 98.30 \\
\hline $\mathrm{Si}^{\mathrm{a}}$ & 1.82 & 1.90 & 1.94 & 1.78 & 1.90 & 1.95 & 1.83 & 2.04 & 1.94 & 1.89 & 1.94 & 1.94 & 1.79 \\
\hline $\mathrm{Al}^{\mathrm{IV}}$ & 0.15 & 0.10 & 0.05 & 0.22 & 0.10 & 0.05 & 0.17 & 0.00 & 0.06 & 0.07 & 0.02 & 0.06 & 0.17 \\
\hline $\mathrm{Al}^{\mathrm{VI}}$ & 0.00 & 0.02 & 0.00 & 0.05 & 0.02 & 0.01 & 0.01 & 0.02 & 0.02 & 0.00 & 0.00 & 0.00 & 0.00 \\
\hline $\mathrm{Fe}^{+3}$ & 0.14 & 0.05 & 0.06 & 0.09 & 0.05 & 0.26 & 0.11 & 0.00 & 0.03 & 0.20 & 0.11 & 0.04 & 0.18 \\
\hline $\mathrm{Ti}$ & 0.05 & 0.04 & 0.02 & 0.06 & 0.03 & 0.01 & 0.05 & 0.00 & 0.01 & 0.05 & 0.02 & 0.03 & 0.05 \\
\hline $\mathrm{Cr}$ & 0.01 & 0.00 & 0.02 & 0.00 & 0.00 & 0.00 & 0.01 & 0.00 & 0.01 & 0.00 & 0.00 & 0.00 & 0.00 \\
\hline $\mathrm{Mg}$ & 0.78 & 0.75 & 0.88 & 0.71 & 0.74 & 0.15 & 0.66 & 1.79 & 1.03 & 0.61 & 0.26 & 0.70 & 0.78 \\
\hline $\mathrm{Fe}^{+2}$ & 0.06 & 0.21 & 0.06 & 0.14 & 0.25 & 0.54 & 0.18 & 0.13 & 0.23 & 0.17 & 0.70 & 0.38 & 0.06 \\
\hline $\mathrm{Mn}$ & 0.01 & 0.01 & 0.00 & 0.01 & 0.01 & 0.03 & 0.02 & 0.00 & 0.01 & 0.02 & 0.04 & 0.02 & 0.00 \\
\hline $\mathrm{Ni}$ & 0.01 & 0.00 & 0.00 & 0.00 & 0.00 & 0.00 & 0.01 & 0.00 & 0.00 & 0.00 & 0.00 & 0.00 & 0.00 \\
\hline $\mathrm{Ca}$ & 0.94 & 0.88 & 0.91 & 0.89 & 0.86 & 0.76 & 0.88 & 0.01 & 0.64 & 0.86 & 0.85 & 0.80 & 0.94 \\
\hline $\mathrm{Na}$ & 0.04 & 0.04 & 0.05 & 0.05 & 0.04 & 0.24 & 0.07 & 0.01 & 0.02 & 0.14 & 0.06 & 0.03 & 0.03 \\
\hline $\mathrm{O}$ & 6.00 & 6.00 & 6.00 & 6.00 & 6.00 & 6.00 & 6.00 & 6.05 & 6.00 & 6.00 & 6.00 & 6.00 & 6.00 \\
\hline \multicolumn{14}{|l|}{ Rim } \\
\hline $\mathrm{SiO}_{2}$ & 47.99 & 43.74 & 46.56 & 46.29 & 50.06 & 49.47 & 46.32 & 58.93 & 48.22 & 48.42 & 48.43 & 51.02 & 48.08 \\
\hline $\mathrm{TiO}_{2}$ & 2.22 & 3.89 & 3.49 & 2.84 & 1.11 & 0.58 & 3.18 & 0.04 & 1.72 & 2.46 & 0.50 & 1.01 & 1.56 \\
\hline $\mathrm{Al}_{2} \mathrm{O}_{3}$ & 3.64 & 8.22 & 4.88 & 6.33 & 2.51 & 0.64 & 6.11 & 0.36 & 4.60 & 2.07 & 0.30 & 1.25 & 3.41 \\
\hline $\mathrm{Cr}_{2} \mathrm{O}_{3}$ & 0.36 & 0.00 & 0.04 & 0.00 & 0.02 & 0.00 & 0.33 & 0.17 & 0.40 & 0.00 & 0.00 & 0.00 & 0.15 \\
\hline $\mathrm{Fe}_{2} \mathrm{O}_{3}$ & 5.11 & 5.32 & 4.92 & 3.41 & 2.98 & 12.74 & 4.45 & 0.00 & 3.75 & 8.43 & 2.96 & 1.43 & 5.32 \\
\hline $\mathrm{FeO}$ & 2.23 & 3.86 & 2.41 & 5.47 & 6.80 & 13.16 & 5.34 & 4.30 & 5.52 & 5.40 & 20.79 & 11.74 & 1.91 \\
\hline $\mathrm{MnO}$ & 0.24 & 0.14 & 0.05 & 0.13 & 0.30 & 0.68 & 0.47 & 0.15 & 0.15 & 0.61 & 1.13 & 0.49 & 0.11 \\
\hline $\mathrm{MgO}$ & 13.60 & 11.25 & 12.87 & 11.46 & 13.26 & 2.39 & 10.72 & 35.57 & 13.51 & 9.75 & 4.31 & 12.47 & 14.17 \\
\hline $\mathrm{NiO}$ & 0.23 & 0.00 & 0.05 & 0.00 & 0.03 & 0.00 & 0.30 & 0.08 & 0.00 & 0.00 & 0.00 & 0.00 & 0.00 \\
\hline $\mathrm{CaO}$ & 23.47 & 23.02 & 24.44 & 22.06 & 21.63 & 16.13 & 21.57 & 0.21 & 21.38 & 21.25 & 19.93 & 19.73 & 23.25 \\
\hline $\mathrm{Na}_{2} \mathrm{O}$ & 0.51 & 0.49 & 0.44 & 0.78 & 0.51 & 4.51 & 1.16 & 0.10 & 0.44 & 2.33 & 0.69 & 0.47 & 0.39 \\
\hline Total & 99.61 & 99.93 & 100.17 & 98.77 & 99.21 & 100.29 & 99.95 & 99.89 & 99.71 & 99.71 & 99.05 & 99.62 & 98.36 \\
\hline $\mathrm{Si}^{\mathrm{a}}$ & 1.80 & 1.65 & 1.74 & 1.76 & 1.89 & 1.95 & 1.75 & 2.02 & 1.81 & 1.85 & 1.96 & 1.94 & 1.82 \\
\hline $\mathrm{Al}^{\mathrm{IV}}$ & 0.16 & 0.35 & 0.22 & 0.24 & 0.11 & 0.03 & 0.25 & 0.00 & 0.19 & 0.09 & 0.01 & 0.06 & 0.15 \\
\hline $\mathrm{Al}^{\mathrm{vi}}$ & 0.00 & 0.01 & 0.00 & 0.04 & 0.00 & 0.00 & 0.02 & 0.01 & 0.01 & 0.00 & 0.00 & 0.00 & 0.00 \\
\hline $\mathrm{Fe}^{+3}$ & 0.14 & 0.15 & 0.14 & 0.10 & 0.08 & 0.38 & 0.13 & 0.00 & 0.11 & 0.24 & 0.09 & 0.04 & 0.15 \\
\hline $\mathrm{Ti}$ & 0.06 & 0.11 & 0.10 & 0.08 & 0.03 & 0.02 & 0.09 & 0.00 & 0.05 & 0.07 & 0.02 & 0.03 & 0.04 \\
\hline $\mathrm{Cr}$ & 0.01 & 0.00 & 0.00 & 0.00 & 0.00 & 0.00 & 0.01 & 0.00 & 0.01 & 0.00 & 0.00 & 0.00 & 0.00 \\
\hline $\mathrm{Mg}$ & 0.76 & 0.63 & 0.72 & 0.65 & 0.75 & 0.14 & 0.60 & 1.82 & 0.75 & 0.55 & 0.26 & 0.71 & 0.80 \\
\hline $\mathrm{Fe}^{+2}$ & 0.07 & 0.12 & 0.08 & 0.17 & 0.21 & 0.43 & 0.17 & 0.12 & 0.17 & 0.17 & 0.70 & 0.37 & 0.06 \\
\hline $\mathrm{Mn}$ & 0.01 & 0.00 & 0.00 & 0.00 & 0.01 & 0.02 & 0.02 & 0.00 & 0.00 & 0.02 & 0.04 & 0.02 & 0.00 \\
\hline $\mathrm{Ni}$ & 0.01 & 0.00 & 0.00 & 0.00 & 0.00 & 0.00 & 0.01 & 0.00 & 0.00 & 0.00 & 0.00 & 0.00 & 0.00 \\
\hline $\mathrm{Ca}$ & 0.94 & 0.93 & 0.98 & 0.90 & 0.87 & 0.68 & 0.87 & 0.01 & 0.86 & 0.83 & 0.86 & 0.80 & 0.94 \\
\hline $\mathrm{Na}$ & 0.04 & 0.04 & 0.03 & 0.06 & 0.04 & 0.34 & 0.08 & 0.01 & 0.03 & 0.17 & 0.05 & 0.03 & 0.03 \\
\hline $\mathrm{O}$ & 6.00 & 6.00 & 6.00 & 6.00 & 6.00 & 6.00 & 6.00 & 6.03 & 6.00 & 6.00 & 6.00 & 6.00 & 6.00 \\
\hline
\end{tabular}

${ }^{\mathrm{a}}$ Mineral formulae were obtained by normalizing on the basis of four cations

the Si site. Calculated water contents ranged from 0.1 to $0.4 \mathrm{wt} \%$.

There are two large euhedral melanite phenocrysts, both with feathery rims. An unidentified, acicular, NaBa-Ti-silicate phase occurs along the edges of the melanites, contributing to the feathery texture of the rims.

\section{Amphibole and mica}

Kaersutite occurs in two samples: IL93-2 and M93-4. Representative analyses are given in Table 6 . In both samples the crystals are brown, rounded and have heavily oxidized rims and almost identical mineral chemistry. The kaersutite is high in $\mathrm{F}$, with $\mathrm{F}$ occupying up to $1 / 4$ of the hydroxyl site. 
Fig. 2 Core-rim compositions of olivine (plotted using Fo content) and pyroxene (plotted using $\mathrm{Al}^{\mathrm{iv}}$ contents
Olivines:
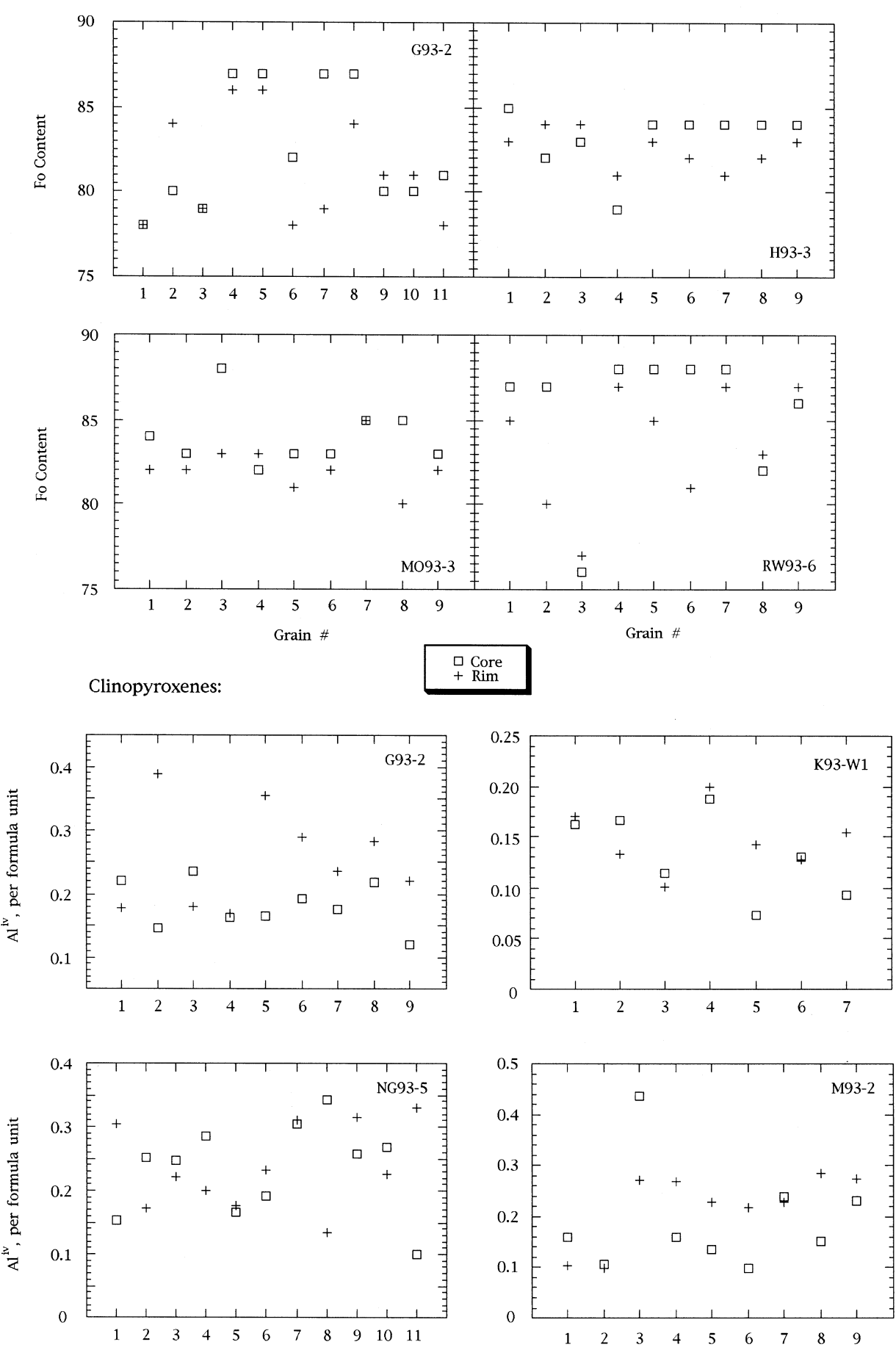

Phlogopite occurs in sample C93-3 as microphenocrysts. Representative analyses are given in Table 6 . The phlogopite is high in $\mathrm{Ba}$ and $\mathrm{F}$, with $\mathrm{F}$ occupying up to $2 / 3$ of the hydroxyl site. Although calculations indicate the phlogopite is high in $\mathrm{Fe}^{3+}$, suggesting the possible presence of significant $\mathrm{Fe}^{3+}$ in the tetrahedral site (tetraferriphlogopite), the phlogopites do not exhibit reverse pleochroism (Cruciani et al. 1995).

\section{Spinels}

Six samples contain titanomagnetite as a phenocryst. Repreentative phenocryst analyses are presented in Table 7. With the exception of four samples, all contain titanomagnetite in the groundmass.

Sample H93-3 contains rare chromite inclusions in olivine. Sample L93-2M does not contain chromite inclusions in olivine, but does have chromite microphe- 
Table 3 Representative nepheline analyses

\begin{tabular}{|c|c|c|c|c|c|c|}
\hline Wt $\%$ oxide & B93-1 & L93-3 & MO93-1 & MR93-2 & RW93-4 & S93-2 \\
\hline $\mathrm{SiO}_{2}$ & 42.80 & 43.56 & 41.11 & 41.49 & 42.75 & 41.74 \\
\hline $\mathrm{Al}_{2} \mathrm{O}_{3}$ & 32.61 & 32.35 & 32.54 & 32.00 & 32.85 & 33.67 \\
\hline $\mathrm{Fe}_{2} \mathrm{O}_{3}$ & 1.19 & 0.84 & 1.96 & 2.56 & 0.74 & 0.94 \\
\hline $\mathrm{MgO}$ & 0.01 & 0.08 & 0.08 & 0.08 & 0.03 & 0.01 \\
\hline $\mathrm{BaO}$ & 0.00 & 0.28 & 0.00 & 0.05 & 0.00 & 0.14 \\
\hline $\mathrm{CaO}$ & 0.30 & 0.19 & 0.04 & 0.03 & 0.26 & 0.03 \\
\hline $\mathrm{SrO}$ & 0.04 & 0.18 & 0.00 & 0.01 & 0.00 & 0.03 \\
\hline $\mathrm{Na}_{2} \mathrm{O}$ & 15.74 & 17.74 & 14.98 & 15.39 & 16.60 & 16.72 \\
\hline $\mathrm{K}_{2} \mathrm{O}$ & 6.64 & 3.89 & 8.62 & 8.04 & 6.25 & 6.05 \\
\hline Total & 99.33 & 99.11 & 99.32 & 99.65 & 99.47 & 99.33 \\
\hline $\mathrm{Si}^{\mathrm{a}}$ & 1.04 & 1.05 & 1.01 & 1.02 & 1.04 & 1.02 \\
\hline $\mathrm{Al}$ & 0.93 & 0.92 & 0.95 & 0.93 & 0.94 & 0.97 \\
\hline $\mathrm{Fe}^{+3}$ & 0.02 & 0.02 & 0.04 & 0.05 & 0.01 & 0.02 \\
\hline $\mathrm{Mg}$ & 0.00 & 0.00 & 0.00 & 0.00 & 0.00 & 0.00 \\
\hline $\mathrm{Ba}$ & 0.00 & 0.00 & 0.00 & 0.00 & 0.00 & 0.00 \\
\hline $\mathrm{Ca}$ & 0.01 & 0.00 & 0.00 & 0.00 & 0.01 & 0.00 \\
\hline $\mathrm{Sr}$ & 0.00 & 0.00 & 0.00 & 0.00 & 0.00 & 0.00 \\
\hline $\mathrm{Na}$ & 0.74 & 0.83 & 0.72 & 0.73 & 0.78 & 0.79 \\
\hline $\mathrm{K}$ & 0.21 & 0.12 & 0.27 & 0.25 & 0.19 & 0.19 \\
\hline $\mathrm{O}$ & 4.00 & 4.00 & 4.00 & 4.00 & 4.00 & 4.00 \\
\hline
\end{tabular}

${ }^{\text {a }}$ Mineral formulae were obtained by normalizing on the basis of 4 oxygens

Table 4 Representative feldspar analyses

\begin{tabular}{lccccc}
\hline $\mathrm{Wt} \%$ oxide & L93-2 & K93-W3 & MD93-4 & NG93-5 & NG93-6 \\
\hline $\mathrm{SiO}_{2}$ & 55.52 & 55.89 & 54.26 & 52.58 & 66.82 \\
$\mathrm{Al}_{2} \mathrm{O}_{3}$ & 25.91 & 25.16 & 27.28 & 28.90 & 18.44 \\
$\mathrm{Fe}_{2} \mathrm{O}_{3}$ & 0.65 & 0.72 & 0.83 & 0.55 & 0.53 \\
$\mathrm{MgO}$ & 0.13 & 0.14 & 0.15 & 0.04 & 0.09 \\
$\mathrm{BaO}$ & 0.37 & 0.03 & 0.38 & 0.00 & 0.49 \\
$\mathrm{CaO}$ & 8.53 & 9.00 & 10.75 & 11.47 & 0.35 \\
$\mathrm{SrO}$ & 0.61 & 0.63 & 0.57 & 0.52 & 0.19 \\
$\mathrm{Na}{ }_{2} \mathrm{O}$ & 6.32 & 6.13 & 5.25 & 4.74 & 8.13 \\
$\mathrm{~K}_{2} \mathrm{O}$ & 0.50 & 0.66 & 0.32 & 0.27 & 5.14 \\
$\mathrm{Total}$ & 98.40 & 98.48 & 99.64 & 99.04 & 100.10 \\
$\mathrm{Si}$ & 2.56 & 2.57 & 2.48 & 2.41 & 2.99 \\
$\mathrm{Al}$ & 1.41 & 1.37 & 1.47 & 1.56 & 0.97 \\
$\mathrm{Fe}$ & 0.02 & 0.02 & 0.03 & 0.02 & 0.02 \\
$\mathrm{Ba}$ & 0.01 & 0.01 & 0.01 & 0.00 & 0.01 \\
$\mathrm{Ca}$ & 0.42 & 0.44 & 0.53 & 0.56 & 0.02 \\
$\mathrm{Sr}$ & 0.02 & 0.02 & 0.02 & 0.01 & 0.00 \\
$\mathrm{Na}$ & 0.56 & 0.55 & 0.46 & 0.42 & 0.71 \\
$\mathrm{~K}$ & 0.03 & 0.04 & 0.02 & 0.02 & 0.29 \\
$\mathrm{Mg}$ & 0.01 & 0.01 & 0.01 & 0.00 & 0.00 \\
$\mathrm{O}$ & 8.00 & 8.00 & 8.00 & 8.00 & 8.00 \\
\hline
\end{tabular}

${ }^{\text {a }}$ Mineral formulae were obtained by normalizing on the basis of 8 oxygens

nocrysts. A representative analysis is presented in Table 7. Sample MD93-4 contains a chromite inclusion in an enstatite grain.

\section{Whole rock chemistry}

The whole rock compositions of the Tanzanian samples are given in Table 8 . There are many different procedures for assigning rock names, and we have chosen one based on chemical composition rather than modal or normative composition. Rock names have been assigned based on total alkalis vs $\mathrm{SiO}_{2}$ after Le Bas (1986) (Table 9). The samples span the range of 38 to $50 \mathrm{wt} \% \mathrm{SiO}_{2}$, with two more evolved samples at 57 and $64 \mathrm{wt} \% \mathrm{SiO}_{2}$. The $\mathrm{MgO}$ concentrations progress from 0.5 to $10 \mathrm{wt} \%$; two samples with 15 and $17 \mathrm{wt} \% \mathrm{MgO}$ probably have accumulated olivine. The $\mathrm{K}_{2} \mathrm{O}(0.8$ to $5.2 \mathrm{wt} \%)$ spans the range expected for the alkali olivine basalt series (Irvine and Baragar 1971). Trace elements do not show a systematic variation with $\mathrm{SiO}_{2}$ because the different primary magmas began their evolution with varying $\mathrm{SiO}_{2}$ concentrations. With decreasing $\mathrm{MgO}$ content, $\mathrm{SiO}_{2}, \mathrm{Al}_{2} \mathrm{O}_{3}, \mathrm{~K}_{2} \mathrm{O}$ and $\mathrm{Na}_{2} \mathrm{O}$ increase. High $\mathrm{Ni}$ concentrations (in excess of $400 \mathrm{ppm}$ ) support the petrographic evidence for accumulation of olivine in MD93-4 and L93-2M.

The trace element patterns of the most primitive Tanzanian lavas are similar to those of ocean island basalt (OIB) (Paslick et al. 1995). The Ba/Nb ratios of the majority of the samples are between 5 and 10, within the range for OIB. The trace element patterns of the more evolved Tanzanian samples show some characteristics of fractionation. These include enrichment in $\mathrm{Nb}$ and $\mathrm{Zr}$, and depletion in $\mathrm{P}$ and Ti dure to fractionation of apatite, pyroxene and titanomagnetite.

\section{Isotopic compositions}

The $\mathrm{Sr}, \mathrm{Nd}$, and $\mathrm{Pb}$ isotopic compositions have been determined on 25 whole rock powders, and nepheline and clinopyroxene mineral separates from five of the samples (Table 9). Clinopyroxene and nepheline phenocrysts were chosen for separation because they are the most abundant and least altered. The $\mathrm{Sr}, \mathrm{Nd}$ and $\mathrm{Pb}$ isotopic compositions of the whole rock samples are all within the field of data for Tanzanian samples measured by Paslick et al. (1995) (Fig. 3a, b).

The $\mathrm{Sr}, \mathrm{Nd}$, and $\mathrm{Pb}$ isotopic compositions of clinopyroxene and nepheline mineral separates are generally displaced from the whole rock isotopic compositions for the same sample (Table 9). This is illustrated in Fig. 4, in which the data for each mineral separate and respective whole rock are plotted, together with estimated analytical uncertainties. All separates of clinopyroxene and nepheline have $\mathrm{Pb}$ that is considerably less radiogenic than that in the whole rocks (Figs. 4, 5). The greatest difference in ${ }^{206} \mathrm{~Pb} /{ }^{204} \mathrm{~Pb}$ is 20.94 (whole rock) vs 19.10 (clinopyroxene) for sample H93-3. The nephelines all display lesser amounts of $\mathrm{Pb}$ isotopic disequilibrium but the differences are still significant, the smallest being 19.77 (whole rock) vs 19.37 (nepheline) for sample B931. The clinopyroxenes also have $\mathrm{Sr}$ that is less radiogenic and $\mathrm{Nd}$ that is more radiogenic than the respective whole rocks (Figs. 4, 5). The nephelines show little if any difference with whole rock values in $\mathrm{Nd}$ and $\mathrm{Sr}$ isotopic composition (Table 9, Fig. 4). However, the ${ }^{87} \mathrm{Sr} /{ }^{86} \mathrm{Sr}$ ratios of two aliquots of a nepheline separate from B93-1 are close, but not within error of each other. In general, 
Table 5 Representative titanite and perovskite analyses

\begin{tabular}{|c|c|c|c|c|c|c|c|c|c|c|}
\hline \multirow[t]{2}{*}{ and perovskite analyses } & \multirow[t]{2}{*}{ Wt\% oxide } & \multicolumn{6}{|c|}{ Titanite } & \multicolumn{3}{|c|}{ Perovskite } \\
\hline & & B93-1 & L93-3 & MO93-1 & MO93-5 & MR93.2 & S93-2 & MO93-1 & MO93-5 & MR93-2 \\
\hline & $\mathrm{Nb}_{2} \mathrm{O}_{5}$ & 0.97 & 0.27 & 0.14 & 0.15 & 0.27 & 0.20 & 0.86 & 1.09 & 1.12 \\
\hline & $\mathrm{SiO}_{2}$ & 29.87 & 30.13 & 30.38 & 30.08 & 30.28 & 30.16 & 0.01 & 0.02 & 0.00 \\
\hline & $\mathrm{TiO}_{2}$ & 34.39 & 35.08 & 36.87 & 36.29 & 35.80 & 35.33 & 55.34 & 52.32 & 54.95 \\
\hline & $\mathrm{ZrO}_{2}$ & 0.42 & 0.45 & 0.66 & 0.89 & 0.52 & 0.09 & 0.00 & 0.01 & 0.00 \\
\hline & $\mathrm{ThO}_{2}$ & 0.00 & 0.00 & 0.00 & 0.03 & 0.03 & 0.00 & 0.13 & 0.21 & 0.32 \\
\hline & $\mathrm{Al}_{2} \mathrm{O}_{3}$ & 0.92 & 1.74 & 0.16 & 0.13 & 0.21 & 1.03 & 0.02 & 0.03 & 0.04 \\
\hline & $\mathrm{Fe}_{2} \mathrm{O}_{3}$ & 2.18 & 1.61 & 2.09 & 2.04 & 2.44 & 1.83 & 0.43 & 0.79 & 0.53 \\
\hline & $\mathrm{MgO}$ & 0.00 & 0.03 & 0.04 & 0.03 & 0.04 & 0.01 & 0.00 & 0.00 & 0.01 \\
\hline & $\mathrm{CaO}$ & 26.89 & 28.44 & 27.25 & 27.00 & 27.17 & 28.21 & 36.30 & 34.40 & 33.05 \\
\hline & $\mathrm{FeO}$ & 0.00 & 0.00 & 0.00 & 0.00 & 0.00 & 0.00 & 0.00 & 0.00 & 0.00 \\
\hline & $\mathrm{MnO}$ & 0.06 & 0.00 & 0.04 & 0.03 & 0.06 & 0.00 & 0.08 & 0.12 & 0.09 \\
\hline & $\mathrm{SrO}$ & 0.17 & 0.08 & 0.66 & 0.42 & 0.21 & 0.00 & 0.76 & 1.23 & 0.85 \\
\hline & $\mathrm{BaO}$ & 0.01 & 0.06 & 0.11 & 0.03 & 0.02 & 0.12 & 0.12 & 0.05 & 0.03 \\
\hline & $\mathrm{Na}_{2} \mathrm{O}$ & 0.10 & 0.08 & 0.29 & 0.27 & 0.26 & 0.10 & 1.04 & 1.50 & 1.57 \\
\hline & $\mathrm{Y}_{2} \mathrm{O}_{3}$ & 0.01 & 0.02 & 0.04 & 0.01 & 0.07 & 0.03 & 0.02 & 0.03 & 0.05 \\
\hline & $\mathrm{La}_{2} \mathrm{O}_{3}$ & 0.14 & 0.02 & 0.00 & 0.03 & 0.05 & 0.10 & 0.71 & 1.22 & 1.52 \\
\hline & $\mathrm{Ce}_{2} \mathrm{O}_{3}$ & 0.26 & 0.23 & 0.04 & 0.00 & 0.00 & 0.16 & 1.51 & 2.42 & 3.24 \\
\hline & $\mathrm{Pr}_{2} \mathrm{O}_{3}$ & 0.14 & 0.00 & 0.09 & 0.00 & 0.10 & 0.00 & 0.09 & 0.31 & 0.30 \\
\hline & $\mathrm{Nd}_{2} \mathrm{O}_{3}$ & 0.18 & 0.10 & 0.01 & 0.06 & 0.04 & 0.05 & 0.66 & 0.76 & 1.16 \\
\hline & $\mathrm{Sm}_{2} \mathrm{O}_{3}$ & 0.05 & 0.06 & 0.08 & 0.04 & 0.03 & 0.00 & 0.13 & 0.15 & 0.22 \\
\hline & $\mathrm{Gd}_{2} \mathrm{O}_{3}$ & 0.00 & 0.18 & 0.00 & 0.08 & 0.01 & 0.04 & 0.00 & 0.06 & 0.03 \\
\hline & $\mathrm{F}$ & 0.50 & 0.47 & 0.64 & 0.44 & 0.46 & 0.92 & 0.18 & 0.25 & 0.25 \\
\hline & Total & 97.26 & 99.05 & 99.58 & 98.04 & 98.07 & 98.36 & 98.40 & 96.96 & 99.32 \\
\hline & $\mathrm{O} \equiv \mathrm{F}$ & -0.21 & -0.20 & -0.27 & -0.19 & -0.20 & -0.39 & -0.08 & -0.11 & -0.10 \\
\hline & $\mathrm{H}_{2} \mathrm{O}$ & 0.28 & 0.35 & 0.24 & 0.22 & 0.29 & 0.24 & 0.33 & 0.70 & 0.19 \\
\hline & Total & 97.33 & 99.20 & 99.55 & 98.07 & 98.17 & 98.22 & 98.65 & 97.56 & 99.40 \\
\hline & $\mathrm{Si}^{\mathrm{a}}$ & 0.994 & 0.976 & 0.984 & 0.994 & 0.997 & 0.973 & 0.000 & 0.000 & 0.000 \\
\hline & $\mathrm{Al}$ & 0.036 & 0.067 & 0.006 & 0.005 & 0.008 & 0.039 & 0.001 & 0.001 & 0.001 \\
\hline & $\mathrm{Fe}^{3+}$ & 0.055 & 0.039 & 0.051 & 0.051 & 0.061 & 0.044 & 0.011 & 0.021 & 0.014 \\
\hline & $\mathrm{Ti}$ & 0.861 & 0.855 & 0.898 & 0.902 & 0.887 & 0.858 & 0.971 & 0.937 & 0.975 \\
\hline & $\mathrm{Zr}$ & 0.007 & 0.007 & 0.010 & 0.014 & 0.008 & 0.001 & 0.000 & 0.000 & 0.000 \\
\hline & $\mathrm{Mg}$ & 0.000 & 0.002 & 0.002 & 0.001 & 0.002 & 0.000 & 0.000 & 0.000 & 0.000 \\
\hline & $\mathrm{Fe}^{2+}$ & 0.000 & 0.000 & 0.000 & 0.000 & 0.000 & 0.000 & 0.000 & 0.000 & 0.000 \\
\hline & $\mathrm{Ca}$ & 0.959 & 0.987 & 0.945 & 0.955 & 0.958 & 0.975 & 0.907 & 0.877 & 0.835 \\
\hline & $\mathrm{Na}$ & 0.007 & 0.005 & 0.018 & 0.017 & 0.016 & 0.006 & 0.047 & 0.069 & 0.072 \\
\hline & $\mathrm{Mn}$ & 0.002 & 0.000 & 0.001 & 0.001 & 0.002 & 0.000 & 0.002 & 0.002 & 0.002 \\
\hline & $\mathrm{Sr}$ & 0.003 & 0.002 & 0.012 & 0.008 & 0.004 & 0.000 & 0.010 & 0.017 & 0.012 \\
\hline & $\mathrm{Ba}$ & 0.000 & 0.001 & 0.001 & 0.000 & 0.000 & 0.002 & 0.001 & 0.000 & 0.000 \\
\hline & $\mathrm{Th}$ & 0.000 & 0.000 & 0.000 & 0.000 & 0.000 & 0.000 & 0.001 & 0.001 & 0.002 \\
\hline & $\mathrm{Nb}$ & 0.015 & 0.004 & 0.002 & 0.002 & 0.004 & 0.003 & 0.009 & 0.012 & 0.012 \\
\hline & Y & 0.000 & 0.000 & 0.001 & 0.000 & 0.001 & 0.000 & 0.000 & 0.000 & 0.001 \\
\hline & $\mathrm{La}$ & 0.002 & 0.000 & 0.000 & 0.000 & 0.001 & 0.001 & 0.006 & 0.011 & 0.013 \\
\hline & $\mathrm{Ce}$ & 0.003 & 0.003 & 0.000 & 0.000 & 0.000 & 0.002 & 0.013 & 0.021 & 0.028 \\
\hline & $\operatorname{Pr}$ & 0.002 & 0.000 & 0.001 & 0.000 & 0.001 & 0.000 & 0.001 & 0.003 & 0.003 \\
\hline & $\mathrm{Nd}$ & 0.002 & 0.001 & 0.000 & 0.001 & 0.000 & 0.001 & 0.005 & 0.006 & 0.010 \\
\hline & $\mathrm{Sm}$ & 0.001 & 0.001 & 0.001 & 0.000 & 0.000 & 0.000 & 0.001 & 0.001 & 0.002 \\
\hline & $\mathrm{Gd}$ & 0.000 & 0.002 & 0.000 & 0.001 & 0.000 & 0.000 & 0.000 & 0.001 & 0.000 \\
\hline & Sum Ca site: & 0.994 & 1.006 & 0.984 & 0.987 & 0.989 & 0.990 & 1.003 & 1.022 & 0.991 \\
\hline & $\mathrm{F}$ & 0.053 & 0.048 & 0.065 & 0.046 & 0.048 & 0.093 & 0.013 & 0.019 & 0.019 \\
\hline . & $\mathrm{O}$ & 4.878 & 4.850 & 4.851 & 4.887 & 4.878 & 4.784 & 2.968 & 2.935 & 2.976 \\
\hline of cations for titanite (3) & $\mathrm{OH}$ & 0.061 & 0.075 & 0.051 & 0.049 & 0.064 & 0.051 & 0.051 & 0.111 & 0.030 \\
\hline
\end{tabular}

${ }^{a}$ Mineral formulae were obtained by normalizing on the basis of cations for titanite (3) and perovskite (2) the $\mathrm{Pb}$ isotopic compositions show the most consistent and significant differences between whole rock and mineral separate pairs. The isotopic compositional differences found in this study are similar to those found by Simonetti and Bell (1994a, b) for whole rock/clinopyroxene mineral separate pairs in nephelinites from Napak volcano in eastern Uganda. However, in that case the sign (or "direction") of the disequilibrium is more variable. Such disequilibrium appears to be normal rather than exceptional in alkaline volcanic rocks in which analyses have been made of mineral separates (Cortini and van Calsteren 1985). It is therefore reasonable to infer that alkaline magmas normally evolve in open systems.

\section{Discussion}

Disequilibrium

The thermodynamically based MELT program (Ghiorso and Sack 1995) can be used to obtain the composition of 
Table 6 Representative analyses of amphibole, melanite and phlogopite

\begin{tabular}{|c|c|c|c|c|c|c|c|c|c|c|c|}
\hline & \multicolumn{4}{|c|}{ Amphibole } & & \multicolumn{4}{|c|}{ Melanite } & \multirow[t]{3}{*}{ Phlogopite } & \multirow[b]{3}{*}{ C93-3 } \\
\hline & \multirow[t]{2}{*}{ L93-2 } & \multirow[t]{2}{*}{ L93-2 } & \multirow[t]{2}{*}{ M93-4 } & \multirow[t]{2}{*}{ M93-4 } & & \multicolumn{2}{|c|}{8 cations: } & \multicolumn{2}{|c|}{3 cations: } & & \\
\hline & & & & & & B93-1 & B93-1 & B93-1 & B93-1 & & \\
\hline Wt\% oxide & core & rim & core & rim & Wt\% oxide & core & rim & core & rim & Wt\% oxide & \\
\hline $\mathrm{SiO}_{2}$ & 40.04 & 40.17 & 38.54 & 38.16 & $\mathrm{SiO}_{2}$ & 30.15 & 30.30 & 30.15 & 30.30 & $\mathrm{SiO}_{2}$ & 32.74 \\
\hline $\mathrm{TiO}_{2}$ & 5.38 & 5.30 & 5.76 & 6.46 & $\mathrm{TiO}_{2}$ & 8.93 & 8.67 & 8.93 & 8.67 & $\mathrm{TiO}_{2}$ & 17.18 \\
\hline $\mathrm{Al}_{2} \mathrm{O}_{3}$ & 10.73 & 10.58 & 12.06 & 11.89 & $\mathrm{ZrO}_{2}$ & 0.47 & 0.62 & 0.47 & 0.62 & $\mathrm{Al}_{2} \mathrm{O}_{3}$ & 12.14 \\
\hline $\mathrm{Cr}_{2} \mathrm{O}_{3}$ & 0.18 & 0.20 & 0.15 & 0.20 & $\mathrm{Al}_{2} \mathrm{O}_{3}$ & 1.91 & 1.90 & 1.91 & 1.90 & $\mathrm{Cr}_{2} \mathrm{O}_{3}$ & 0.01 \\
\hline $\mathrm{Fe}_{2} \mathrm{O}_{3}$ & 1.66 & 0.51 & 0.67 & 0.00 & $\mathrm{Fe}_{2} \mathrm{O}_{3}$ & 24.19 & 22.83 & 24.19 & 23.33 & $\mathrm{Fe}_{2} \mathrm{O}_{3}$ & 5.80 \\
\hline $\mathrm{FeO}$ & 11.42 & 12.45 & 11.75 & 13.77 & $\mathrm{Cr}_{2} \mathrm{O}_{3}$ & 0.00 & 0.00 & 0.00 & 0.00 & $\mathrm{FeO}$ & 4.21 \\
\hline $\mathrm{MnO}$ & 0.33 & 0.36 & 0.41 & 0.47 & $\mathrm{Y}_{2} \mathrm{O}_{3}$ & 0.12 & 0.10 & 0.12 & 0.10 & $\mathrm{MnO}$ & 0.11 \\
\hline $\mathrm{MgO}$ & 12.31 & 12.24 & 12.45 & 10.40 & $\mathrm{~V}_{2} \mathrm{O}_{3}$ & 0.24 & 0.18 & 0.24 & 0.18 & $\mathrm{MgO}$ & 14.77 \\
\hline $\mathrm{BaO}$ & 0.61 & 0.66 & 0.72 & 0.85 & $\mathrm{MgO}$ & 0.49 & 0.44 & 0.49 & 0.44 & $\mathrm{BaO}$ & 5.53 \\
\hline $\mathrm{CaO}$ & 11.01 & 11.24 & 11.52 & 11.24 & $\mathrm{FeO}$ & 0.00 & 0.45 & 0.00 & 0.00 & $\mathrm{CaO}$ & 0.04 \\
\hline $\mathrm{Na}_{2} \mathrm{O}$ & 2.82 & 2.82 & 2.75 & 2.71 & $\mathrm{CaO}$ & 32.15 & 32.43 & 32.15 & 32.43 & $\mathrm{SrO}$ & 0.01 \\
\hline $\mathrm{K}_{2} \mathrm{O}$ & 0.82 & 0.88 & 1.44 & 1.31 & $\mathrm{MnO}$ & 0.53 & 0.49 & 0.53 & 0.49 & $\mathrm{Na}_{2} \mathrm{O}$ & 0.76 \\
\hline $\mathrm{F}^{2}$ & 0.90 & 0.86 & 1.08 & 0.66 & $\mathrm{SrO}$ & 0.00 & 0.00 & 0.00 & 0.00 & $\mathrm{~K}_{2} \mathrm{O}$ & 4.28 \\
\hline $\mathrm{Cl}$ & 0.08 & 0.09 & 0.08 & 0.08 & $\mathrm{Na}_{2} \mathrm{O}$ & 0.26 & 0.24 & 0.26 & 0.24 & $\mathrm{~F}$ & 3.39 \\
\hline $\mathrm{H}_{2} \mathrm{O}$ & 1.54 & 1.56 & 1.46 & 1.36 & $\mathrm{~F}$ & 0.07 & 0.00 & 0.07 & 0.00 & $\mathrm{Cl}$ & 0.03 \\
\hline $\mathrm{O} \equiv \mathrm{F}$ & -0.38 & -0.36 & -0.45 & -0.28 & Total & 99.51 & 98.64 & 99.51 & 98.69 & $\mathrm{H}_{2} \mathrm{O}$ & 0.77 \\
\hline $\mathrm{O} \equiv \mathrm{Cl}$ & -0.02 & -0.02 & -0.02 & -0.02 & $\mathrm{O} \equiv \mathrm{F}$ & 99.48 & 98.64 & 99.48 & 98.69 & $\mathrm{O} \equiv \mathrm{F}$ & -1.43 \\
\hline \multirow[t]{2}{*}{ Total } & 99.43 & 99.54 & 100.37 & 99.27 & $\mathrm{H}_{2} \mathrm{O}$ & & & 0.20 & 0.38 & $\mathrm{H} \equiv \mathrm{Cl}$ & -0.01 \\
\hline & & & & & Total & & & 99.68 & 99.07 & Total & 100.33 \\
\hline \multicolumn{12}{|l|}{$T$ sites $^{\mathrm{a}}$} \\
\hline $\mathrm{Si}$ & 6.03 & 6.06 & 5.80 & 5.86 & $\mathrm{Si}$ & 2.56 & 2.60 & 2.50 & 2.50 & & \\
\hline $\mathrm{Al}^{\mathrm{IV}}$ & 1.90 & 1.88 & 2.14 & 2.14 & $\mathrm{Ti}$ & 0.57 & 0.56 & 0.56 & 0.54 & $\mathrm{Sr}$ & 5.09 \\
\hline M1,2,3 sites & & & & & $\mathrm{Zr}$ & 0.02 & 0.03 & 0.02 & 0.03 & $\mathrm{Al}^{\mathrm{IV}}$ & 2.23 \\
\hline $\mathrm{Al}^{\mathrm{Vi}}$ & 0.00 & 0.00 & 0.00 & 0.02 & $\mathrm{Al}^{\mathrm{IV}}$ & 0.19 & 0.19 & 0.25 & 0.17 & $\mathrm{Fe}^{3+}$ & 0.68 \\
\hline $\mathrm{Ti}$ & 0.61 & 0.60 & 0.65 & 0.75 & $\mathrm{Al}^{\mathrm{VI}}$ & 0.00 & 0.00 & 0.00 & 0.00 & $\mathrm{Al}^{\mathrm{VI}}$ & 0.00 \\
\hline $\mathrm{Cr}$ & 0.02 & 0.02 & 0.02 & 0.02 & $\mathrm{Fe}^{3+}$ & 1.55 & 1.47 & 1.51 & 1.45 & $\mathrm{Ti}$ & 2.01 \\
\hline $\mathrm{Fe}^{3+}$ & 0.19 & 0.06 & 0.08 & 0.00 & $\mathrm{Cr}$ & 0.00 & 0.00 & 0.00 & 0.00 & $\mathrm{Cr}$ & 0.00 \\
\hline $\mathrm{Fe}^{2+}$ & 1.44 & 1.57 & 1.48 & 1.77 & $\mathrm{Y}$ & 0.01 & 0.00 & 0.01 & 0.00 & $\mathrm{Fe}^{2+}$ & 0.55 \\
\hline $\mathrm{Mn}$ & 0.04 & 0.05 & 0.05 & 0.06 & $\mathrm{~V}$ & 0.02 & 0.01 & 0.02 & 0.01 & $\mathrm{Mn}$ & 0.01 \\
\hline $\mathrm{Mg}$ & 2.76 & 2.75 & 2.79 & 2.38 & $\mathrm{Mg}$ & 0.06 & 0.06 & 0.06 & 0.05 & $\mathrm{Mg}$ & 3.43 \\
\hline M4 site & & & & & $\mathrm{Fe}^{2+}$ & 0.00 & 0.03 & 0.00 & 0.00 & $\mathrm{Ba}$ & 0.34 \\
\hline $\mathrm{Ba}$ & 0.04 & 0.04 & 0.04 & 0.05 & $\mathrm{Ca}$ & 2.93 & 2.98 & 2.86 & 2.87 & $\mathrm{Ca}$ & 0.01 \\
\hline $\mathrm{Ca}$ & 1.78 & 1.82 & 1.86 & 1.85 & $\mathrm{Mn}$ & 0.04 & 0.04 & 0.04 & 0.03 & $\mathrm{Sr}$ & 0.00 \\
\hline $\mathrm{Na}$ & 0.22 & 0.18 & 0.14 & 0.15 & $\mathrm{Sr}$ & 0.00 & 0.00 & 0.00 & 0.00 & $\mathrm{Na}$ & 0.23 \\
\hline A site & & & & & $\mathrm{Na}$ & 0.04 & 0.04 & 0.04 & 0.04 & $\mathrm{~K}$ & 0.85 \\
\hline $\mathrm{Na}$ & 0.60 & 0.64 & 0.66 & 0.66 & $\mathrm{O}$ & 11.99 & 12.00 & 11.80 & 11.56 & A site & 1.42 \\
\hline K & 0.16 & 0.17 & 0.28 & 0.26 & $\mathrm{~F}$ & 0.02 & 0.00 & 0.02 & 0.00 & $\mathrm{O}$ & 20.00 \\
\hline Sum & 0.76 & 0.81 & 0.93 & 0.92 & $\mathrm{OH}$ & & & 0.04 & 0.08 & $\mathrm{O}$ & 1.19 \\
\hline $\mathrm{O}$ & 22.00 & 22.00 & 22.00 & 22.00 & & & & & & $\mathrm{~F}$ & 1.67 \\
\hline $\mathrm{F}$ & 0.43 & 0.41 & 0.51 & 0.32 & & & & & & $\mathrm{Cl}$ & 0.01 \\
\hline $\mathrm{Cl}$ & 0.02 & 0.02 & 0.02 & 0.02 & & & & & & $\mathrm{OH}$ & 0.80 \\
\hline $\mathrm{OH}$ & 1.55 & 1.57 & 1.47 & 1.39 & & & & & & & \\
\hline
\end{tabular}

${ }^{a}$ Mineral formulae were obtained by normalizing on the basis of cations for melanite (8), pholgopite (14) and amphibole (13)

olivine precipitating from a basaltic liquid assuming that the whole rock chemistry is representative of the melt and that olivine is the liquidus phase. In addition, the ferric/ferrous ratio of the liquid must be known. This program has been used to evaluate whether the observed olivine phenocryst compositions could be in equilibrium with the liquid represented by the whole rock chemistry. For 4 of the 15 samples containing olivine, the observed olivine compositions were too fayalitic to have crystallized from the bulk liquid at any $f_{\mathrm{O}_{2}}$ greater than the iron-wüstite buffer (i.e., the liquid would have been in equilibrium with metallic iron, an unreasonable assumption given the presence of ferrian spinels).
We infer from the mineral chemistry that the remaining 11 samples also contain xenocrystic olivine. This is illustrated in some samples by a complete lack of zoning; minerals crystallizing from a magma should be normally zoned (rims enriched in $\mathrm{Fe}$ ) due to the enrichment of $\mathrm{Fe}$ over $\mathrm{Mg}$ in the melt during crystallization. Disequilibrium is illustrated in other samples by the presence of both normally and reversely zoned olivines, and by the presence of two populations of olivine with different major element compositions (see Fig. 2). Sample MD93-4 is an excellent example of a rock containing two different populations of olivine; one is unzoned and has core compositions of $\mathrm{Fo}_{93}$, whereas the second popula- 
Table 7 Representative titanomagnetite analyses

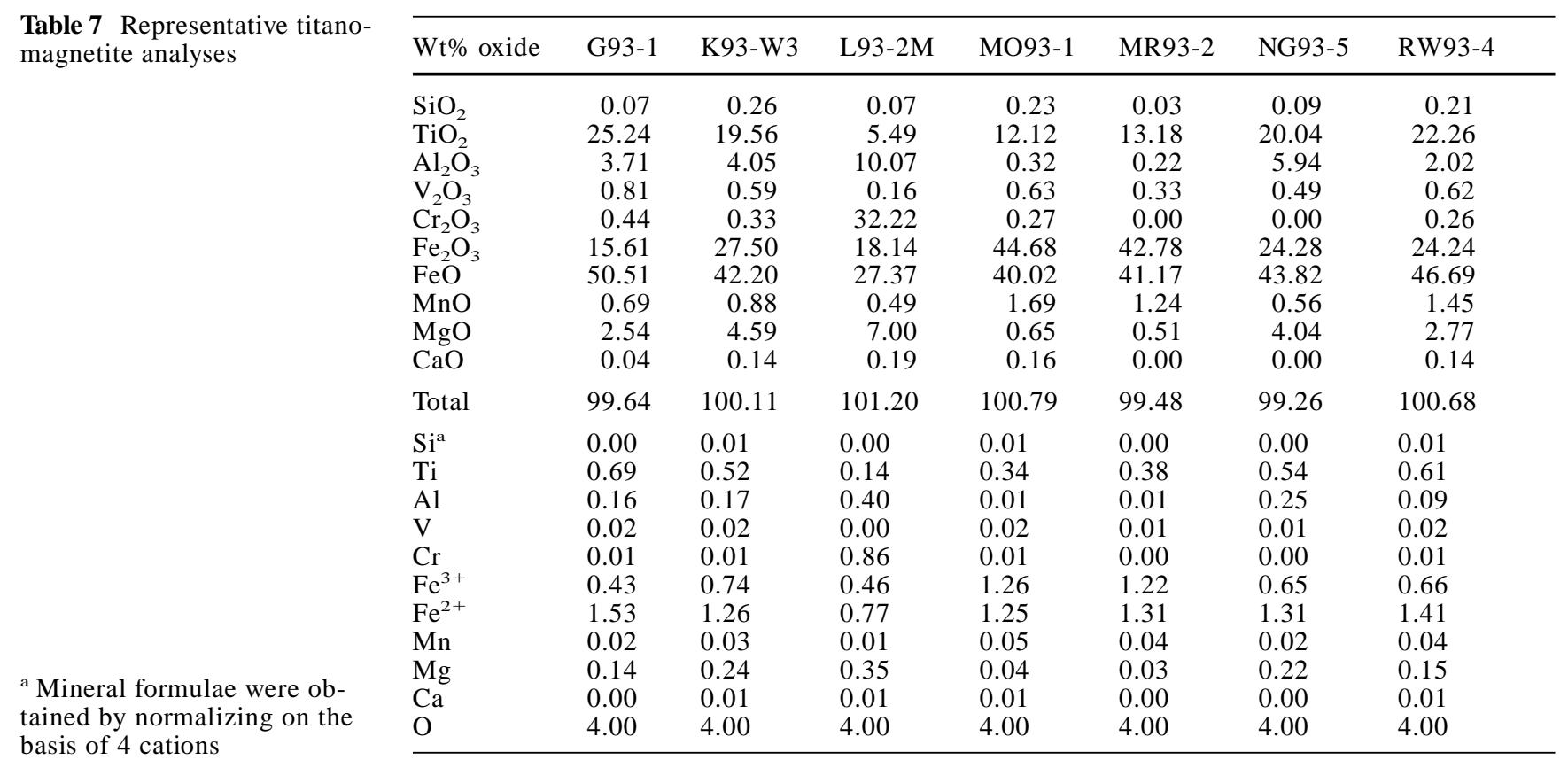

${ }^{a}$ Mineral formulae were obtained by normalizing on the basis of 4 cations

Table 8 Major and trace element chemistry of northern Tanzanian lavas ${ }^{\mathrm{a}}$

\begin{tabular}{|c|c|c|c|c|c|c|c|c|c|c|c|c|c|c|}
\hline ample & B93-1 & G93-1 & G93-2 & K93-W3 & L93-3 & M93-2 & M93-4 & MO93-1 & MU93-3 & MO9. & MR9. & $N G Y$ & $N G$ & \\
\hline $\mathrm{SiO}_{2}$ & 47.09 & 43.08 & 43.45 & 46.68 & 52.11 & 47.60 & 49.46 & 43.70 & 41.93 & 43.53 & 43.97 & 44.94 & 64.97 & 38.11 \\
\hline $\mathrm{Al}_{2} \mathrm{O}_{3}$ & 18.74 & 12.16 & 12.25 & 14.36 & 19.46 & 17.57 & 17.21 & 12.93 & 11.74 & 17.53 & 18.11 & 15.7 & 13.40 & 7.64 \\
\hline $\mathrm{Fe}_{2} \mathrm{O}_{3}$ & 5.40 & 14.72 & 14.50 & 12.64 & 6.22 & 9.30 & 9.28 & 12.15 & 12.33 & 9.39 & 10.00 & 13.67 & 7.46 & 13.21 \\
\hline $\mathrm{MgO}$ & 1.60 & 9.12 & 9.11 & 6.38 & 0.41 & 2.61 & 2.68 & 2.22 & 7.23 & 1.28 & 1.41 & 5.72 & 0.47 & 17.08 \\
\hline $\mathrm{CaO}$ & 7.05 & 11.07 & 11.18 & 8.62 & 4.03 & 7.06 & 6.03 & 7.26 & 11.65 & 4.28 & 4.41 & 10.3 & 0.98 & 12.44 \\
\hline $\mathrm{Na}_{2} \mathrm{O}$ & 8.35 & 2.59 & 2.63 & 4.56 & 9.61 & 8.15 & 7.63 & 8.43 & 4.19 & 12.17 & 9.45 & 3.29 & 6.24 & 2.45 \\
\hline $\mathrm{K}_{2} \mathrm{O}$ & 5.29 & 1.03 & 1.05 & 2.10 & 4.34 & 4.27 & 3.83 & 2.70 & 1.70 & 6.16 & 4.50 & 0.96 & 4.57 & 1.51 \\
\hline $\mathrm{TiO}_{2}$ & 0.62 & 3.28 & 3.23 & 2.73 & 0.62 & 2.43 & 2.31 & 3.21 & 3.01 & 2.13 & 2.36 & 3.3 & 0.68 & 3.92 \\
\hline $\mathrm{MnO}$ & 0.25 & 0.22 & 0.21 & 0.20 & 0.18 & 0.20 & 0.2 & 0.44 & 0.20 & 0.33 & 0.33 & 0.18 & 0.23 & 0.21 \\
\hline $\mathrm{P}_{2} \mathrm{O}_{5}$ & 0.21 & 0.73 & 0.71 & 0.62 & 0.08 & 0.56 & 0.64 & 0.52 & 0.72 & 0.52 & 0.37 & 0.79 & 0.07 & 0.84 \\
\hline LOI & 4.38 & 1.49 & 1.64 & 0.88 & 1.56 & 0.05 & -0.29 & 5.84 & 4.36 & 0.64 & 4.44 & 0.63 & 0.50 & 2.19 \\
\hline Total & 98.99 & 99.49 & 99.96 & 99.76 & 98.62 & 99.81 & 98.98 & 99.40 & 99.07 & 97.96 & 99.35 & 99.48 & 99.57 & 99.60 \\
\hline $\mathrm{Nb}$ & 54.6 & 65.5 & 52.4 & 107.2 & 166.4 & 225.7 & 216.1 & 275.3 & 56.4 & 311.2 & 314.2 & 64.5 & 124.3 & 143.8 \\
\hline $\mathrm{Zr}$ & 168.1 & 303.7 & 164.8 & 297.8 & 355.2 & 515.7 & 471.6 & 887.9 & 302.9 & 646.4 & 523.0 & 145.6 & 299.4 & 417.3 \\
\hline Y & 29.0 & 32.5 & 28.3 & 29.0 & 31.4 & 37.1 & 37.7 & 62.2 & 44.9 & 55.1 & 54.9 & 25.4 & 27.4 & 29.1 \\
\hline $\mathrm{Sr}$ & 1168.5 & 818.0 & 1122.2 & 939.3 & 1455.3 & 1709.9 & 1510.0 & 2537.8 & 683.2 & 2213.2 & 1573.0 & 1783.2 & 1403.0 & 1597.9 \\
\hline $\mathrm{Rb}$ & 28.2 & 47.1 & 25.3 & 48.1 & 103.4 & 111.3 & 109.8 & 109.7 & 33.3 & 178.8 & 138.4 & 22.9 & 52.3 & 43.6 \\
\hline Th & 8.7 & 9.4 & 7.8 & 13.5 & 29.6 & 22.7 & 22.2 & 12.7 & 8.5 & 25.1 & 26.7 & 8.7 & 8.5 & 13.5 \\
\hline $\mathrm{Pb}$ & 3.2 & 9.9 & 4.5 & 7.8 & 28.4 & 19.0 & 16.2 & 17.7 & 4.1 & 14.6 & 13.3 & 5.8 & 3.5 & 7.7 \\
\hline $\mathrm{Zn}$ & 112.4 & 98.8 & 110.6 & 115.5 & 150.5 & 131.7 & 121.4 & 303.9 & 124.5 & 235.7 & 238.7 & 103.7 & 107.0 & 108.2 \\
\hline $\mathrm{Cu}$ & 59.8 & 25.7 & 63.5 & 79.0 & 17.9 & 33.5 & 27.8 & 179.7 & 24.8 & 92.3 & 108.9 & 66.2 & 174.4 & 138.7 \\
\hline $\mathrm{Ni}$ & 172.9 & 21.1 & 180.8 & 73.5 & 1.9 & 5.6 & 5.5 & 5.2 & 21.5 & 3.9 & 6.6 & 40.8 & 97.1 & 423.3 \\
\hline $\mathrm{Cr}$ & 277.2 & 19.8 & 291.5 & 120.1 & 0.0 & 0.0 & 0.0 & 0.0 & 18.6 & 0.2 & 0.0 & 50.3 & 202.7 & 1024.5 \\
\hline $\mathrm{Ce}$ & 100.7 & 137.2 & 95.9 & 145.1 & 202.9 & 235.7 & 251.2 & 171.7 & 105.1 & 171.5 & 220.0 & 121.6 & 158.2 & 150.7 \\
\hline $\mathrm{Nd}$ & 48.4 & 62.3 & 51.3 & 63.6 & 49.1 & 84.2 & 84.7 & 60.4 & 61.2 & 56.5 & 76.3 & 55.5 & 69.6 & 73.8 \\
\hline $\mathrm{La}$ & 42.9 & 67.0 & 37.1 & 82.5 & 138.1 & 147.5 & 143.3 & 106.7 & 52.8 & 126.1 & 152.4 & 64.3 & 70.5 & 79.5 \\
\hline $\mathrm{V}$ & 245.7 & 150.3 & 246.9 & 221.5 & 24.0 & 160.7 & 115.1 & 296.7 & 186.7 & 176.2 & 194.6 & 233.8 & 270.1 & 277.0 \\
\hline $\mathrm{Ba}$ & 512.4 & 717.3 & 493.8 & 788.6 & 1382.5 & 1393.1 & 1499.8 & 4170.3 & 590.8 & 2803.0 & 3196.5 & 676.8 & 1165.8 & 963.7 \\
\hline $\mathrm{Sc}$ & 16.7 & 12.8 & 16.8 & 11.2 & 0.0 & 5.2 & 2.0 & 0.0 & 17.8 & 0.0 & 0.0 & 9.1 & 13.7 & 31.7 \\
\hline
\end{tabular}

${ }^{a}$ Whole rock compositions for C93-3, H93-3, K93-1, K93-W1, K93-W2, L93-2, MD93-4, MD93-7, OE93-4, RW93-4, RW93-6 and S93-2 published by Paslick et al. (1995)

tion is normally zoned with core compositions of $\mathrm{Fo}_{88}$ to $\mathrm{Fo}_{90}$.

Because pyroxene compositions are more complex than those of olivines it is difficult to determine whether pyroxene phenocrysts are in equilibrium with the whole rock chemistry on the basis of mineral composition. However, many of the samples in this study contain both normally and reversely zoned pyroxenes, suggesting that at least some of the crystals are xenocrysts (Fig. 2). Microprobe analysis of nepheline and plagioclase show a 
Table 9 Isotopic compositions of northern Tanzanian lavas

\begin{tabular}{|c|c|c|c|c|c|c|c|}
\hline Sample & $\begin{array}{l}\text { Lithology/ } \\
\text { (phase) }\end{array}$ & ${ }^{87} \mathrm{Sr} /{ }^{86} \mathrm{Sr}$ & ${ }^{143} \mathrm{Nd} /{ }^{144} \mathrm{Nd}$ & $\varepsilon_{\mathrm{Nd}}$ & ${ }^{206} \mathrm{Ph} /{ }^{204} \mathrm{~Pb}$ & ${ }^{207} \mathrm{~Pb} /{ }^{204} \mathrm{~Pb}$ & ${ }^{208} \mathrm{~Pb} /{ }^{204} \mathrm{~Pb}$ \\
\hline \multicolumn{8}{|l|}{ Burko } \\
\hline B93-1 & Foidite & $0.704559 \pm 14$ & $0.512642 \pm 09$ & $+0.09 \pm 0.18$ & 19.772 & 15.758 & 39.974 \\
\hline B93-1a & (Nepheline) & $0.704640 \pm 20$ & & & 19.236 & 15.646 & 39.399 \\
\hline B93-1b & (Nepheline) & $0.704546 \pm 32$ & & & 19.368 & 15.684 & 39.496 \\
\hline \multicolumn{8}{|l|}{ Gelai } \\
\hline G93-1 & Basanite & $0.704002 \pm 18$ & $0.512703 \pm 20$ & $+1.27 \pm 0.39$ & 20.049 & 15.664 & 39.747 \\
\hline G93-1b & (Pyroxene) & $0.703891 \pm 18$ & $0.512774 \pm 29$ & $+2.67 \pm 0.57$ & 19.385 & 15.648 & 38.893 \\
\hline G93-2 & Basanite & $0.704002 \pm 23$ & $0.512792 \pm 53$ & $+3.01 \pm 1.03$ & 20.112 & 15.707 & 39.879 \\
\hline \multicolumn{8}{|l|}{ Kwaraha } \\
\hline H93-3 ${ }^{\mathrm{a}}$ & Foidite & $0.703529 \pm 17$ & $0.512749 \pm 10$ & $+2.17 \pm 0.20$ & 20.937 & 15.755 & 40.509 \\
\hline H93-3a & (Pyroxene) & $0.703284 \pm 17$ & $0.512787 \pm 16$ & $+2.92 \pm 0.31$ & & & \\
\hline H93-3b & (Pyroxene) & $0.703291 \pm 18$ & $0.512813 \pm 17$ & $+3.42 \pm 0.33$ & 19.097 & 15.624 & 38.620 \\
\hline \multicolumn{8}{|l|}{ Lemagrut } \\
\hline L93-3 & Phonolite & $0.705126 \pm 20$ & $0.512491 \pm 10$ & $-2.87 \pm 0.20$ & 19.360 & 15.630 & 39.675 \\
\hline \multicolumn{8}{|l|}{ Meru } \\
\hline M93-2 & Phonolite & $0.704352 \pm 21$ & $0.512671 \pm 09$ & $+0.66 \pm 0.18$ & 18.830 & 15.518 & 39.099 \\
\hline \multirow{2}{*}{\multicolumn{8}{|c|}{ Mosonik }} \\
\hline & & & & & & & \\
\hline MO93-1 & Foidite & $0.704148 \pm 32$ & $0.512729 \pm 12$ & $+1.78 \pm 0.23$ & & & \\
\hline MO93-3 & Basanite & $0.704049 \pm 18$ & $0.512731 \pm 20$ & $+1.81 \pm 0.39$ & 20.031 & 15.634 & 39.876 \\
\hline MO93-5 & Foidite & $0.704243 \pm 18$ & $0.512701 \pm 18$ & $+1.23 \pm 0.35$ & 19.843 & 15.653 & 39.962 \\
\hline MO93-5a & (Nepheline) & $0.704246 \pm 24$ & & & & & \\
\hline MO93-5b & (Nepheline) & & & & 18.651 & 15.596 & 38.255 \\
\hline MR93-2 & Foidite & $0.704308 \pm 20$ & $0.512682 \pm 12$ & $+0.86 \pm 0.23$ & 19.912 & 15.679 & 40.061 \\
\hline \multicolumn{8}{|l|}{ Ngorongoro } \\
\hline NG93-5 & Basanite & $0.704237 \pm 21$ & $0.512715 \pm 09$ & $+1.51 \pm 0.18$ & 19.844 & 15.680 & 39.923 \\
\hline NG93-6 & Trachyte & $0.704865 \pm 17$ & $0.512772 \pm 25$ & $+2.61 \pm 0.49$ & 20.126 & 15.822 & 40.189 \\
\hline \multicolumn{8}{|l|}{ Sadiman } \\
\hline S93-2 & Foidite & $0.705428 \pm 15$ & $0.512483 \pm 18$ & $-3.02 \pm 0.35$ & 20.079 & 15.759 & 39.998 \\
\hline S93-2a & (Nepheline) & $0.705408 \pm 22$ & $0.512486 \pm 15$ & $-2.95 \pm 0.29$ & 19.254 & 15.664 & 38.729 \\
\hline \multicolumn{8}{|c|}{ Isolated Cones } \\
\hline L93-2M & Foidite & $0.7038734 \pm 14$ & $0.512795 \pm 12$ & $+3.06 \pm 0.23$ & 19.878 & 15.628 & 39.780 \\
\hline
\end{tabular}

${ }^{a}$ Data from Paslick et al. 1995

complete lack of zoning, implying that these minerals may also be xenocrysts.

The isotopic analyses of the bulk mineral separate/ whole rock pairs supports the conclusion of a xenocrystic origin for nepheline and pyroxene (Figs. 3, 4, 5). The whole rock/mineral separate pair of analyses from the most evolved sample (S93-2) show disequilibrium only in the $\mathrm{Pb}$ isotopic composition, whereas the $\mathrm{Sr}$ and $\mathrm{Nd}$ isotopic compositions of the whole rock and nepheline separate are identical within error. Sample H93-3, with $13 \mathrm{wt} \% \mathrm{MgO}$ and $39 \mathrm{wt} \% \mathrm{SiO}_{2}$ (see Paslick et al. 1995 for complete whole rock chemistry) shows greater disequilibrium in $\mathrm{Sr}, \mathrm{Nd}$ and $\mathrm{Pb}$ isotopes. This may indicate that for S93-2 the $\mathrm{Sr}$ and $\mathrm{Nd}$ isotopes in the xenocrysts were able to equilibrate during time spent in the magma chamber.

The more likely explanation for the consistent difference in $\mathrm{Pb}$ but not $\mathrm{Nd}$ and $\mathrm{Sr}$ isotopic ratios between the mineral separate and the whole rock is simply the much lower concentration of $\mathrm{Pb}$. We have modeled the change in $\mathrm{Nd}$ and $\mathrm{Pb}$ isotopic compositions as a result of mixing between two end-members: lower crustal xenoliths (from Lashaine, see Fig. 1) were chosen as the crustal endmember, and the sample with the most radiogenic $\mathrm{Nd}$ and Pd was chosen as representative of the primary melt (see Paslick et al. 1995 for complete whole rock chemistry and isotopic compositions of sample BD216; this sample has $6 \mathrm{wt} \% \mathrm{MgO}$ and $37.5 \mathrm{wt} \% \mathrm{SiO}_{2}$ ). This model combines binary mixing equations for two elements with different isotope ratios, in this case $\mathrm{Nd}$ and $\mathrm{Pd}$, for two end-members. The results of this model were published previously (Fig. 5 in Paslik et al. 1995). The value of this ratio using the two end-members specified above is 4.4 , and much of the isotopic data clusters near this line. The calculations are all for $(\mathrm{Nd} / \mathrm{Pb})_{\mathrm{c}}<(\mathrm{Nd} / \mathrm{Pb}) \mathrm{m}$, (i.e., for $\mathrm{Pb}$ concentrations higher in the crust than in the primary magma). It is apparent from these calculations that the isotopic ratio of $\mathrm{Pb}$ in the resulting liquid is affected well before there is any significant change in the isotopic ratio of $\mathrm{Nd}$. This difference is even more extreme for $\mathrm{Sr}$ and $\mathrm{Pb}$ because the $\mathrm{Sr}$ concentrations in these lavas range from 500 to $2500 \mathrm{ppm}$.

\section{Possible sources of the xenocrysts}

\section{Crustal contamination}

There are several possible explanations for the disequilibrium seen in both the chemical and isotopic compositions of the minerals. The most obvious is crustal contamination of the magma during residence in a magma 

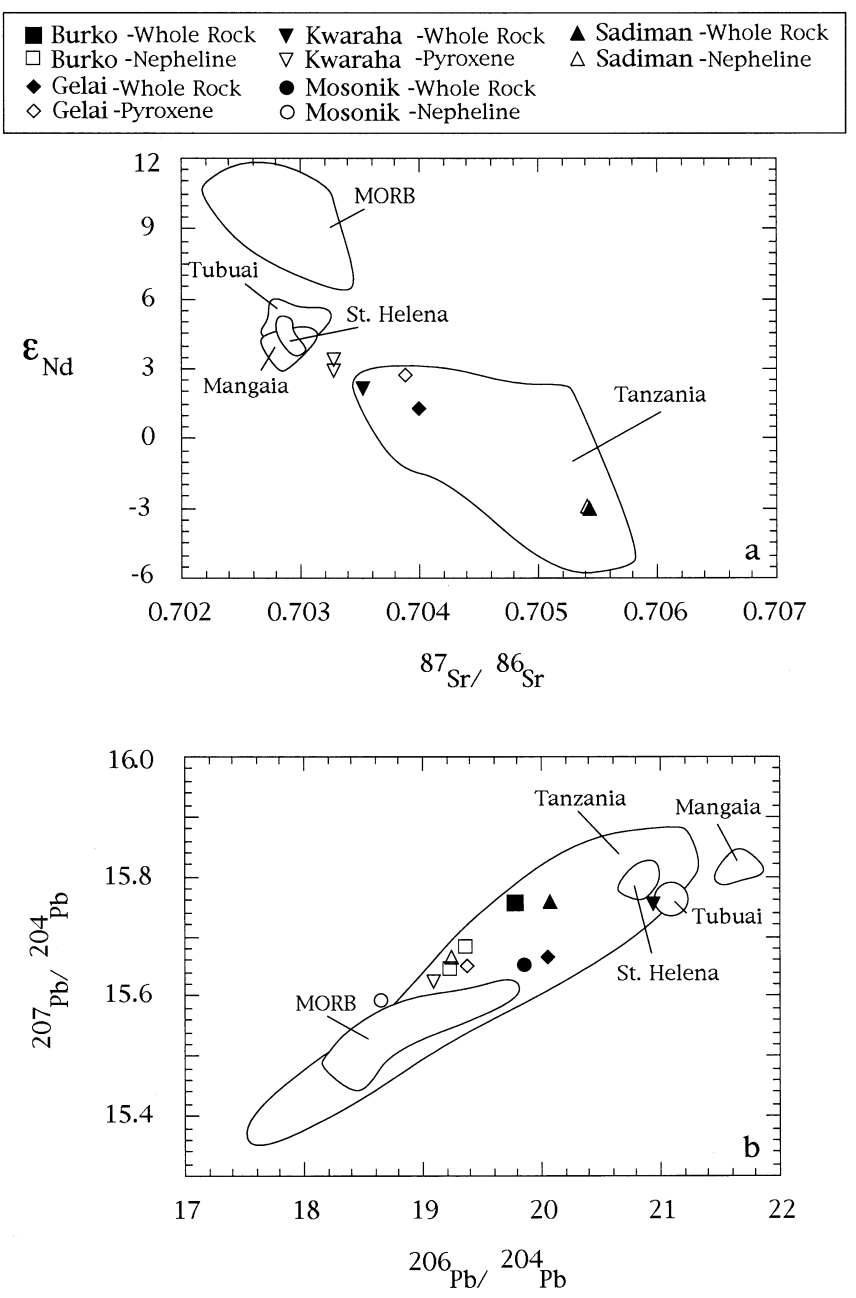

Fig. 3a $\varepsilon_{\mathrm{Nd}}$ vs ${ }^{87} \mathrm{Sr} /{ }^{86} \mathrm{Sr}$ for whole rock analyses. $\mathbf{b}{ }^{206} \mathrm{~Pb} /{ }^{204} \mathrm{~Pb}$ vs ${ }^{207} \mathrm{~Pb} /{ }^{204} \mathrm{~Pb}$ for whole rock analyses. Data: Mangaia and Tubaii (Hauri and Hart 1993), MORB (Newsom et al. 1986), St. Helena (Sun 1980), Tanzanian field (Paslick et al. 1995)

chamber. The samples in this study are all silica undersaturated and it is likely that they have experienced some olivine and/or pyroxene fractionation. Using the MELTS program (Ghiorso and Sack 1995) we have modeled fractionation concurrent with assimilation of a granitic upper crustal component (hornblende granite, Adirondacks, New York, Buddington 1957 p. 293, analysis 1). These calculations illustrate that it is not possible to rule out 5 to $10 \%$ assimilation of an upper crustal component on the basis of major element chemistry.

We have modeled the effect of assimilation-fractional crystallization (AFC) on $\mathrm{Pb}$ isotopic ratios (DePaolo 1981, Paslick et al. 1995). The vector produced from contamination by upper crust is significantly steeper than the trend of the $\mathrm{Pb}$ isotopic data. The $\mathrm{Pb}$ isotopic data for the less radiogenic Tanzanian samples are compatible with lower crustal contamination.

The xenocryst phases identified in the northern Tanzanian samples do not appear to be consistent with what is known of the mineralogical composition of the crust in northern Tanzania. The exposed basement rock in northern Tanzania consists of metamorphosed plutonic rocks and greenstone belts (Coomer and Robertson 1974; Bell and Dodson 1981; Shackleton 1986). Any minerals scavenged from northern Tanzanian country rock would be significantly out of chemical and isotopic equilibrium with the magma. Petrographically, preserved crustal xenocrysts would be easy to identify: minerals such as quartz and feldspar would have obvious reaction rims. The xenocrysts in this study do not appear petrographically to be out of equilibrium with the host melt: only with the additional information given by mineral chemistry and isotopic data has disequilibrium been identified. None of the samples contain quartz and only one sample contains a plagioclase $\left(\mathrm{An}_{45}\right)$ that appears texturally to be out of equilibrium. the minerals that are xenocrystic in these lavas are olivine, pyroxene, plagioclase and nepheline. Mineral chemistry indicates that the xenocrysts precipitated from an alkali basaltic magma. There is no evidence that the lavas scavenged minerals from the exposed schists and gneisses.

Mafic granulite lower crustal xenoliths (garnet-plagioclase clinopyroxenites) brought to the surface by the Lashaine tuff cone near Monduli (see Fig. 1) represent another possible of xenocrysts (Jones et al. 1983). The composition of the clinopyroxenes in these xenoliths is similar to that of the clinopyroxenes in this study, with the exception of significantly lower $\mathrm{TiO}_{2}$ concentrations (Jones et al. 1983). Plagioclase in the xenoliths is significantly less anorthite-rich than that in the Tanzanian samples, with the exception of the single plagioclase grain that occurs in MD93-4. Garnet is an additional common mineral in the xenoliths and does not occur in the Tanzanian lavas. The differences in mineral chemistry suggest that the mafic granulite lower crust is not the source of the xenocrysts in these samples. In support of this, $\mathrm{Pb}$ isotopic whole rock data from the mafic granulites are significantly less radiogenic than the mineral separates in this study: ${ }^{206} \mathrm{~Pb} /{ }^{204} \mathrm{~Pb}$ ranges between 15.8 and 17.5 in the xenoliths (Cohen et al. 1984) vs a range of 18.60 to 19.82 in the Tanzanian mineral separates (Table 9).

\section{Mantle contamination}

An alternative possibility is that the disequilibrium reflects the addition of components derived from the mantle. Sample MD93-4 appears to contain mantle derived olivine $\left(\mathrm{Fo}_{93}\right)$; sample L93-2M contains some olivine phenocrysts with chromite inclusions that are $\mathrm{Fo}_{90}$. These olivines may have been incorporated in the mantle. In most samples, however, the compositions of olivine and pyroxene do not support a mantle origin. With the exception of the samples mentioned above, olivines in this study range up to $\mathrm{Fo}_{81}$. These olivines are simply too Fe-rich to be mantle xenocrysts. Clinopyroxenes in these samples have significantly less $\mathrm{Cr}$ and significantly more Al than expected for mantle clinopyroxe- 


$$
{ }^{87} \mathrm{Sr} /{ }^{86} \mathrm{Sr}
$$
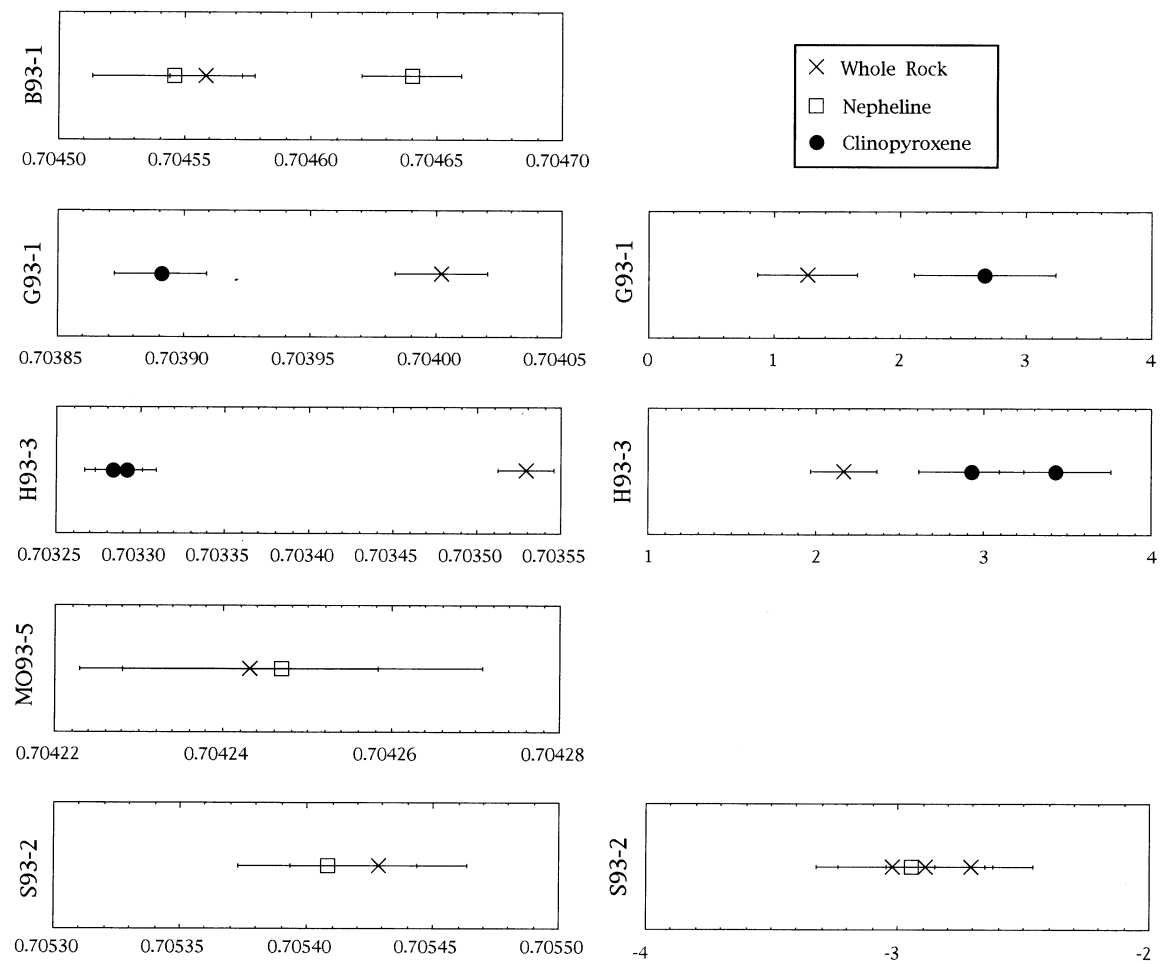

$\varepsilon_{\mathrm{Nd}}$
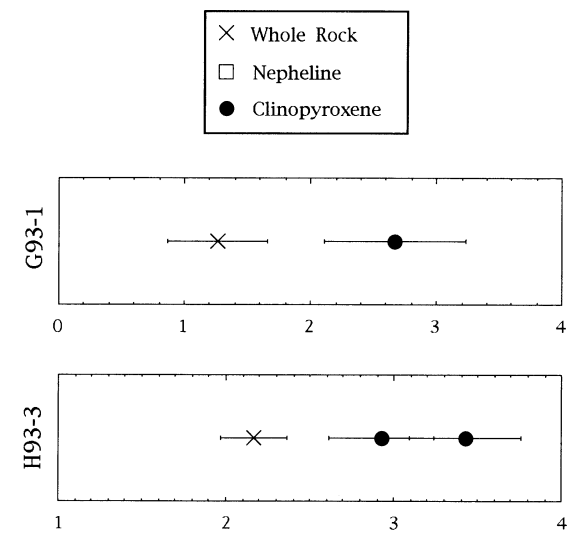

Fig. $4 \mathrm{Sr}, \mathrm{Nd}$, and $\mathrm{Pb}$ isotopic data for each mineral separate and respective whole rock together with estimated analytical uncertainties

nes. The similarity of xenocrystic clinopyroxenes from Napak volcano in Uganda to magmatic clinopyroxenes from other alkaline complexes, in addition to the difference in composition (higher $\mathrm{Al}$, total $\mathrm{Fe}$ and $\mathrm{Ti}$ ) between Napak clinopyroxenes and Cr-bearing clinopyroxenes from East Africa believed to be mantle xenocrysts, led Simonetti and Bell (1994b) to conclude that xenocrystic clinopyroxenes from Napak were not derived from the mantle.

\section{Magma mixing}

Mixing of two basaltic magmas has been suggested previously to explain disequilibrium in alkali basalts (Duda and Schmincke 1985; Simonetti and Bell 1994b). In the Eiffel province, heterogeneous clinopyroxenes are made up of an Fe-rich core and a normally zoned mantle of comagmatic titanian augite (Duda and Schmincke 1985). The authors interpret the pyroxenes as illustrating polybaric magma evolution: primitive alkali basalt rose through the upper mantle fractionating Al-augite in route; the magma then stagnated near the crust/mantle boundary, fractionating Fe-rich clinopyroxenes. This differentiated magma was subsequently mixed with new pulses of primitive alkali basalt, precipitating augite rims on the Fe-rich pyroxenes.
Xenocrysts from Napak in Uganda show complex zoning in single grains, suggesting that they were crystallizing from a melt undergoing continual contamination by a second liquid (Simonetti and Bell 1994b). The xenocrysts in this study do not show either the complex zoning or the diopsidic reaction rims seen in the Napak clinopyroxenes. Both the texture and mineral chemistry of the Tanzanian samples are more consistent with partial assimilation of a solidified basaltic magma. The mineral chemistry of some of the samples in this study may require partial melting and assimilation of more than two batches of magma. Clinopyroxene and olivine populations seen in samples NG93-5 and G93-2 respectively, appear to have a variety of core compositions. It is possible that the conduits feeding volcanism in Tanzania have had more than one batch of basaltic magma solidify before reaching the surface.

Magma mixing, in the form of a second liquid assimilating a small part of an initial solidified basalt, provides a possible explanation for the consistently less radiogenic $\mathrm{Pb}$ isotopic compositions of the mineral separates. If the solidified magma had assimilated lower crust as it cooled, it would be expected to have less radiogenic $\mathrm{Pb}$ than the primary melt. However, this type of crustal contamination should also be apparent as a decrease in the ${ }^{143} \mathrm{Nd} /{ }^{144} \mathrm{Nd}$ and an increase in the ${ }^{87} \mathrm{Sr} /{ }^{86} \mathrm{Sr}$ of the mineral separates. The $\mathrm{Sr}$ and $\mathrm{Nd}$ isotopic compositions of the nepheline separates are almost all within error of the whole rock isotopic compositions. The clinopyroxenes have consistently less radiogenic $\mathrm{Sr}$ and more radiogenic $\mathrm{Nd}$, the opposite of what is expected from crustal con- 

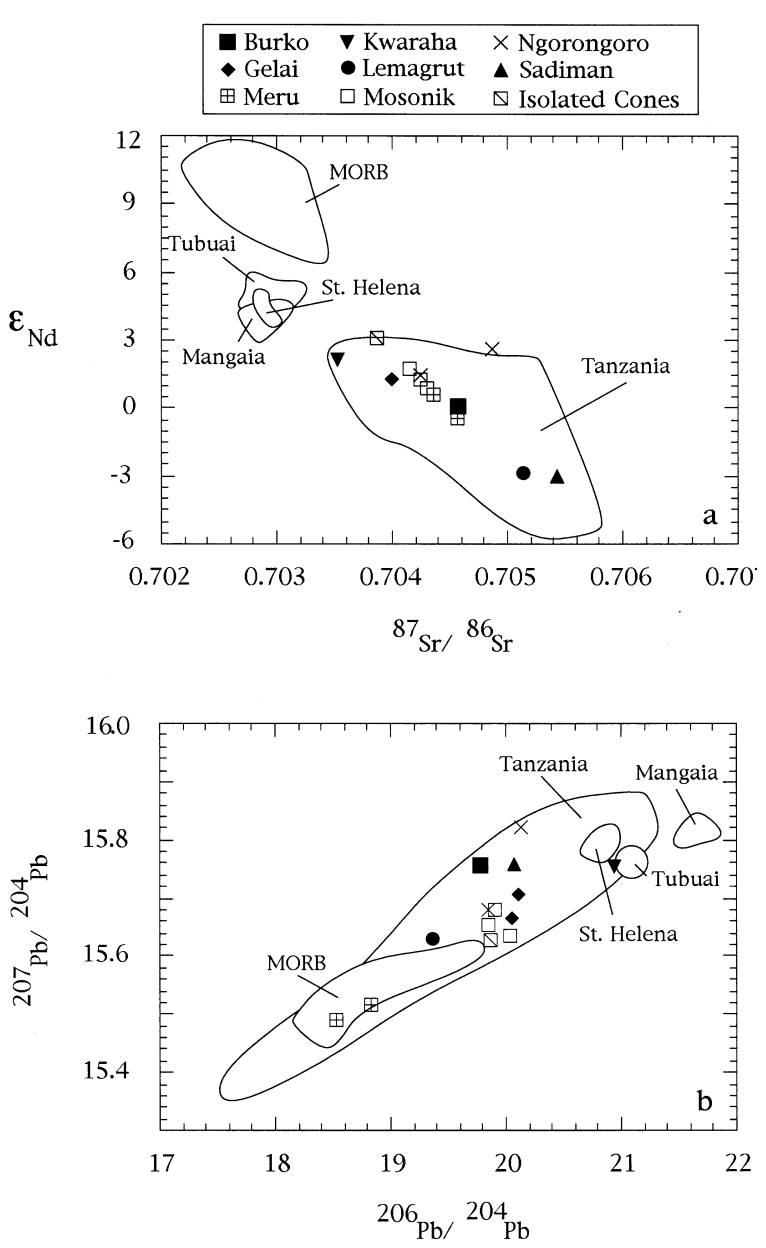

Fig. 5a $\varepsilon{ }^{v s}{ }^{87} \mathrm{Sr} /{ }^{86} \mathrm{Sr}$ for whole rock/mineral pairs. b ${ }^{206} \mathrm{~Pb} /$ ${ }^{204} \mathrm{~Pb} v{ }^{207} \mathrm{~Pb} /{ }^{204} \mathrm{~Pb}$ for whole rock/mineral pairs. Data: Mangaia and Tubaii (Hauri and Hart 1993), MORB (Newsom et al. 1986), St. Helena (Sun 1980), Tanzania field (Paslick et al. 1995)

tamination. It is also possible that the first batch of magma acquired its isotopic composition from a different source, requiring no contamination. Because the $\mathrm{Pb}$ concentrations in these samples are very low (2-8 ppm), $\mathrm{Pb}$ would be one of the first elements to show contamination. The $\mathrm{Sr}$ and $\mathrm{Nd}$ are significantly more abundant (500-2500 and 50-100 ppm respectively) and it would require more assimilation to change the isotopic ratio of these elements. Magma mixing may also explain the fayalitic nature of the olivine xenocrysts. A basaltic melt that has solidified along a conduit in the lower crust may have olivine compositions that are more Fe-rich due to differentiation of the magma during crystallization.

\section{The effect of contamination}

The effect of xenocryst contamination on whole rock measurements has been modeled by calculating the $\mathrm{Pb}$ isotopic composition of the magma before contamination with the xenocrystic material. The ${ }^{206} \mathrm{~Pb} /{ }^{204} \mathrm{~Pb}$ isotopic composition of the pristine magma was calculated

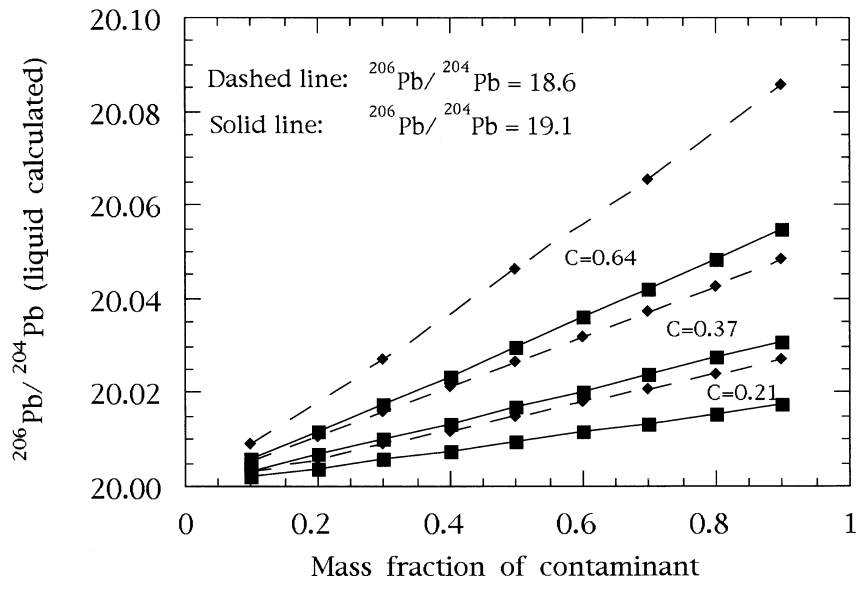

Fig. 6 Calculated lines of the ${ }^{206} \mathrm{~Pb} /{ }^{204} \mathrm{~Pb}$ compositions of magma prior to contamination for various volume fractions of xenocrysts and bulk xenocryst concentration of $\mathrm{Pb}$. Se text for full explanation. Model parameters: ${ }^{206} \mathrm{~Pb} /{ }^{204} \mathrm{~Pb}$ of xenocryst $=18.6$ and $19.1,{ }^{206} \mathrm{~Pb} /{ }^{204} \mathrm{~Pb}$ of whole rock $=20$, concentration of $\mathrm{Pb}$ in $\mathrm{cpx}=1 \mathrm{ppm}$, in ne $=2 \mathrm{ppm}$. Bulk concentration of $\mathrm{Pb}$ calculated by $\mathrm{C}^{\mathrm{T}}=\left(\mathrm{C}_{\mathrm{cpx}} * \mathrm{f}_{\mathrm{cpx}}+\mathrm{C}_{\mathrm{ne}} * \mathrm{f}_{\mathrm{ne}}\right) ; c p x$ clinopyroxene, ne nepheline, $f$ mass fraction of crystals, $C$ concentration

assuming contamination with two different batches of xenocrystic material each with a different value of ${ }^{206} \mathrm{~Pb} /{ }^{204} \mathrm{~Pb}$ (Fig. 6). The isotopic compositions of the xenocrystic material are from a nepheline separate from MO93-5 with the least radiogenic $\mathrm{Pb}$ of the mineral separates $\left({ }^{206} \mathrm{~Pb} /{ }^{204} \mathrm{~Pb}=18.6\right)$ and a clinopyroxene separate from $\mathrm{H} 93-3$ with the average $\mathrm{Pb}$ isotopic composition of the mineral separates $\left({ }^{206} \mathrm{~Pb} /{ }^{204} \mathrm{~Pb}=19.1\right.$, Table 9). Also calculated (although not plotted) is the liquid composition using a contaminant with a ${ }^{206} \mathrm{~Pb} /{ }^{204} \mathrm{~Pb}$ of 17.5 , similar to those values measured for lower crustal granulite xenoliths from Lashaine. We have not measured values as unradiogenic as these in our mineral separate samples, but the calculation illustrates the importance of the isotopic composition of the xenocrystic material. A value of 20 was used in both calculations for the ${ }^{206} \mathrm{~Pb} /{ }^{204} \mathrm{~Pb}$ ratio of the contaminated whole rock. Modal amounts of the minerals (Table 10) were used to calculate the bulk concentration of $\mathrm{Pb}$ in the xenocrysts using the equation $\mathrm{C}_{\mathrm{X}}=\left\{\left(\mathrm{f}_{j} X_{j}\right)+\left(\mathrm{f}_{i} X_{i}\right)\right\}$ (where $\mathrm{f}=$ mass fraction, $X=$ concentration, $i=$ nepheline and $j=$ clinopyroxene and $\mathrm{f}_{i}+\mathrm{f}_{j}=1$ ) for four different samples. The samples used to calculate $\mathrm{C}_{\mathrm{X}}$ were chosen because they have significant amounts of xenocrysts. We have analyzed a clinopyroxene separate (G93-1) and a nepheline separate (MO93-5) for Pb concentration; the measured concentrations are 1 and $2 \mathrm{ppm}$ for clinopyroxene and nepheline respectively.

Isotopic compositions calculated for the magma prior to contamination are plotted in Fig. 6. Three lines, with $\mathrm{C}_{\mathrm{X}}=$ bulk concentration of $\mathrm{Pb}$ in the contaminant, ranging from 1.67 to 1.88, represent four samples (B93-1, L93-3, MR93-2 and S93-2), with varying amounts of modal nepheline and clinopyroxene. For the calculation it was assumed that all the nepheline and clinopyroxene in the samples was xenocrystic (worst case scenario). For sample MR93-2, with 30\% nepheline, the calculated $C_{X}$ 
is 1.88. Complete model parameters are listed in the figure caption (Fig. 6). Although most of the samples in this study contain less than $20 \%$ crystals, calculations were plotted for mass fraction of contaminant ranging up to $90 \%$ to illustrate the change in isotopic composition that might occur from extreme contamination. For $30 \%$ xenocrysts $\left({ }^{206} \mathrm{~Pb} /{ }^{204} \mathrm{~Pb}=18.6\right)$, with a bulk $\mathrm{Pb}$ concentration of 1.88 , the calculated ${ }^{206} \mathrm{~Pb} /{ }^{204} \mathrm{~Pb}$ isotopic ratio of the original magma is $0.37 \%$ higher than the whole rock value. For $30 \%$ xenocrysts with ${ }^{206} \mathrm{~Pb} /{ }^{204} \mathrm{~Pb}=19.1$, the change in the ${ }^{206} \mathrm{~Pb} /{ }^{204} \mathrm{~Pb}$ composition is only $0.3 \%$. For xenocrystic material similar to lower crustal granulites, the change in ${ }^{206} \mathrm{~Pb} /{ }^{204} \mathrm{~Pb}(30 \%$ xenocrysts, $\left.\mathrm{C}_{\mathrm{X}}=0.1 .88\right)$ is $0.5 \%$.

\section{Conclusions}

The isotopic composition of a mafic volcanic rock that may appear petrographically to be in equilibrium is suspect. However, it is important to evaluate the extent of disequilibrium and its effect on whole rock chemistry on a sample by sample basis. For instance, if olivine is the only xenocryst in a sample, the isotopic and $\mathrm{Sr}, \mathrm{Nd}$ and $\mathrm{Pb}$ concentration data may be unaffected since olivine has very low concentrations of these trace elements. The modal abundance of xenocrysts and the isotopic composition of the source of the xenocrysts are two factors that will control the change in the composition of the resulting rock.

The Tanzanian volcanic rocks were not generated in the single magma chamber petrologists define as a closed system. These lavas passed through conduits that had been coated with basalt from previous batches of magma that had stalled and solidified before reaching the surface. The northern Tanzanian province has been active for about million 8 years; the volume of lava produced during that time is not large (Dawson 1992) and the heat flow in this area is not as high as might be expected considering that it is in a rift zone (Nyblade and Pollack 1990). All these factors are consistent with a discontinuous type of volcanism where many batches of magma might solidify on the way to the surface due to lack of heat. Evidence of disequilibrium in mineral chemistry and isotopic composition from additional alkaline provinces (Eiffel, Duda and Schmincke 1985; southern Italy, Cortini and van Calsteren 1985) suggests that generation in an open system is common for alkali basalts.

\section{References}

Bagdasaryan GP, Gerasimovski VI, Polyakov AI, Gukasyan RK (1973) Age of volcanic rocks in the rift zones of East Africa Geochem Int 10:66-71

Baker BH, Crossley R, Goles GG (1978) Tectonic and magmatic evolution of the southern part of the Kenya Rift Valley. Petrology and Geochemistry of Continental rifts. Reidel, Dordrecht, pp 29-50 
Bell K, Dodson MH (1981) The geochronology of the Tanzanian shield. J Geol 89: 109-128

Brown G, Stephen I (1959) A structureal study of iddingsite from New South Wales, Australia. Am Mineral 44: 251-260

Buddington AF (1957) Interrelated Precambrian granitic rocks, Northwest Adirondacks, New York. Geol Soc Amer Bull 68: 291-306

Cahen L, Snelling JJ, Delhal J, Vail JR (1984) The geochronology and evolution of Africa. Clarendon Press, Oxford

Carlson RW, Lugmair GW, MacDougall JD (1981) Columbia River volcanism: the question of mantle heterogeneity or crustal contamination. Geochim Cosmochim Acta 45: 2483-2499

Cohen RS, O’Nions RK, Dawson JB (1984) Isotope geochemistry of xenoliths from East Africa: implications for development of mantle reservoirs and their interaction. Earth Planet Sci Lett 68:209-220

Coomer PG, Robertson DK (1974) Lead isotope study of Archean mineralization areas in Tanzania. J Geol Soc London 130: 449460

Cortini M, van Calsteren PWC (1985) Lead isotope differences between whole-rock and phenocrysts in recent lavas from southern Italy. Nature 314:343-345

Cruciani G, Zanazzi PF, Quartieri S (1995) Tetrahedral ferric iron in phlogopite: XANES and Mossbauer compared to singlecrystal X-ray data. Eur J Mineral 7:255-265

Dawson JB (1992) Neogene tectonics and volcanicity in the North Tanzania sector of the Gregory Rift Valley: contrasts with the Kenya sector. Tectonophysics 204: 81-92

Dawson JB, Smith JV, Steele IM (1994) Trace element distribution between co-existing perovskite, apatite and titanite from Oldoinyo Lengai, Tanzania. Chem Geol 117:285-290

DePaolo DJ (1981) Trace element and isotopic effects of combined wall-rock assimilation and fractional crystallization. Earth Planet Sci Lett 53: 189-202

DePaolo DJ, Wasserburg GJ (1979) Neodymium isotopes in flood basalts from the Siberian Platform and inferences about their mantle sources. Proc Nat Acad Sci USA 76(7):3056-3060

Duda A, Schmincke HU (1985) Polybaric differentiation of alkali basaltic magmas: evidence from green-core clinopyroxenes (Eifel, FRG). Contrib Mineral Petrol 91:340-353

Fitton JG, Dunlop HM (1985) The Cameroon line, West Africa, and its bearing on the origin of oceanic and continental alkali basalt. Earth Planet Sci Lett 72:23-38

Fitton JG, James D, Leeman WP (1991) Basic magmatism association with late Cenozoic extension in the western United States: compositional variations in space and time. J Gophys Res 96: 13693-13711

Ghiorso MS, Sack R (1995) Chemical mass transfer in magmatic processes IV. A revised and internally consistent thermodynamic model for the interpolation and extrapolation of liquidsolid equilibria in magmatic systems at elevated temperatures and pressures. Contrib Mineral Petrol 119:197-212

Halliday AN, Dickin AP, Fallick AE, Fitton JG (1988) Mantle dynamics: a $\mathrm{Nd}, \mathrm{Sr}, \mathrm{Pb}$ and $\mathrm{O}$ isotopic study of the Cameroon line volcanic chain. J Petrol 29: 181-211

Halliday AN, Mahood GA, Holden P, Metz JM, Dempster TJ, Davidson JP (1989) Evidence for long-residence times of rhyolitic magma in the Long Valley magmatic system: the isotopic record in precaldera lavas of Glass Mountain. Earth Planet Sci Lett 94: 274-290

Halliday AN, Davidson JP, Holden P, DeWolf CP, Lee D-C, Fitton JG (1990) Trace-element fractionation in a plume and the origin of HIMU mantle beneath the Cameroon line. Nature 347: 523-528
Hart SR, Gerlach DC, White WM (1986) A possible new Sr-Nd$\mathrm{Pb}$ mantle array and consequences for mantle mixing. Geochim Cosmochim Acta 50: 1551-1557

Hauri EH, Hart SR (1993) Re-Os isotope systematics of HIMU and EMII oceanic island basalts from the south Pacific Ocean. Earch Planet Sci Lett 114:353-371

Hawkesworth CJ, Vollmer R (1979) Crustal contamination versus enriched mantle: ${ }^{143} \mathrm{Nd} /{ }^{144} \mathrm{Nd}$ and ${ }^{87} \mathrm{Sr} /{ }^{86} \mathrm{Sr}$ evidence from the Italian Volcanics. Contrib Mineral Petrol 69: 151-165

Irvine TN, Baragar WRA (1971) A guide to the chemical classification of the common volcanic rocks. Can J Earth Sci 8:523548

Jones AP, Smith JV, Hansen EC, Dawson JB (1983) Metamorphism, partial melting and K-metasomatism of garnet-scapolite-kyanite granulite xenoliths from Lashaine, Tanzania. J Geol 91:143-165

Kempton PD, Fitton JG, Hawkesworth CJ, Ormerod DS (1991) Isotopic and trace element constraints on the composition and evolution of the lithosphere beneath the southwestern United States. Journal of Geophysical Research 96: 13713-13735

Key RM, Charsley TJ, Hackman BD, Wilkinson AF, Rundle CC (1989) Superimposed Upper Proterozoic collision-controlled orogenies in the Mozambique Orogenic Belt of Kenya. Precambrian Research 44: 197-225

Le Bas MJ, LeMaitre RW, Streckeisen A, Zanettin B (1986) A chemical classification of volcanic rocks based on the total alkali-silica diagram. J Petrol 27:745-750

Lum CCL, Leeman WP, Foland KA, Kargel JA, Fitton JG (1989) Isotopic variations in continential basaltic lavas as indicators of mantle heterogeneity: examples from the western U.S. Cordillera. J Geophys Res 94: 7871-7884

Newson HE, White WM, Jochum KP, Hofmann AW (1986) Siderophile and chalcophile element abundances in oceanic basalts, $\mathrm{Pb}$ isotope evolution and growth of the earth's core. Earth Planet Sci Lett 80:299-313

Nyblade NA, Pollack HN (1990) Terrestrial heat flow in east and southern Africa. J Geophys Res 95: 17,371-17,384

Paslick CR, Halliday AN, James D, Dawson JB (1995) Enrichment of the continental lithosphere by OIB melts: itotopic evidence from the volcanic province of nothern Tanzania. Earth Planet Sci Lett 130: 109-126

Peterson TD (1989) Peralkaline nephelinites. I. Comparative petrology of Shombole and Oldoinyo L'engai, East Africa. Contrib Mineral Petrol 101:458-478

Shackleton RM (1986) Precambrian collison tectonics in Africa. In: Coward MP, Ries AC (eds) Collison tectonics. Geol Soc London Spec Pub 19:329-349

Simonetti A, Bell K (1994a) Nd, Pb and Sr isotopic data from the Napak carbonatite-nephelinite centre, eastern Uganda: an example of open-system crystal fractionation. Contrib Mineral Petrol 115:356-366

Simonetti A, Bell K (1994b) Isotopic disequilibrium in clinopyroxenes from nephelinitic lavas. Napak volcano, eastern Uganda. Geol 21:243-246

Spera FJ (1984) Carbon dioxide in petrogenesis III: role of volatiles in the ascent of alkaline magma with special reference to xenolith bearing mafic lavas. Contrib Mineral Petrol 88: 217-233

Sun S-S (1980) Lead isotopic study of young volcanic rocks from mid-ocean ridges, ocean islands and island arcs. Phil Trans $\mathrm{R}$ Soc London A297:409-445 